\title{
Theory of Packaged Entangled States
}

\author{
Rongchao Ma \\ Technical Institute of Physics and Chemistry \\ Chinese Academy of Sciences, Beijing 100190, P. R. China
}

Received 21 July 2017

Accepted 18 September 2017

Published 22 November 2017

\begin{abstract}
The quantum entanglement that include every physical properties of particles would be important for both theoretical and applied physics. Here, we theoretically show that a particleantiparticle pair can form the so-called packaged entangled states which encapsulate all the necessary physical quantities for completely identifying the particles. The particles in the packaged entangled states exhibit unusual properties. Thereafter, we proposed the possible protocol for teleporting the entire quantum state of a particle (or an antiparticle) to an arbitrarily large distance and the transfer of new entangled states from a particle pair to another particle pair. Finally, we presented a new interpretation to the matter-antimatter asymmetry of the observable universe.
\end{abstract}

Keywords: Packaged entangled state; charge conjugation; particle-antiparticle teleportation; matter-antimatter asymmetry.

\section{Introduction}

An entangled state usually refers to a pure state of a composite system which cannot be expressed as the direct product of the quantum states of its subsystems. ${ }^{1-3}$ In these states, a measurement on one of the particles will immediately change the state of other particles ${ }^{4}$ via the "spooky action at a distance" a no matter how far these particles are spatially separated. The entangled states are important for both fundamental research ${ }^{4-8}$ and applications in quantum information. ${ }^{9}$ A considerable amount of theoretical and technical works have been devoted to the entanglement of one degree of freedom ${ }^{10-20}$ and several degrees of freedom, i.e., hyperentanglement ${ }^{21-25}$ multimode entanglement ${ }^{26-31}$ and entanglement of

a Letter from Einstein to Max Born, 3 March 1947, p158. The Born-Einstein Letters; Correspondence between Albert Einstein and Max and Hedwig Born from 1916 to 1955 (Macmillan Press Ltd., New York, 1971).

This is an Open Access article published by World Scientific Publishing Company. It is distributed under the terms of the Creative Commons Attribution 4.0 (CC-BY) License. Further distribution of this work is permitted, provided the original work is properly cited. 
indistinguishable particles. ${ }^{32-34}$ These entangled states usually encapsulate part of the particles' physical quantities.

However, one may ask whether the particles can form a special entangled state that can package all their physical quantities? More specifically, a particle possesses a number of independent physical quantities (or freedoms), such as charge, baryon number, lepton number, etc. ${ }^{35,36}$ With these physical quantities, one can completely identify the particles. The question is whether all these physical quantities could be packaged as an entirety in the new entangled state. We shall call such an entangled state as a packaged entangled state, which is different to the states of hyperentanglement $^{21-25}$ or multimode entanglement. ${ }^{26-31}$

The packaged entangled states could be used to teleport the entire quantum states or to transmit every intrinsic property of the particles or antiparticles, ${ }^{37}$ instead of just some of their physical properties. On the other hand, the packaged entangled states could be also used to explain the imbalance between baryons and antibaryons produced after the Big Bang. ${ }^{38}$ Finally, the packaged entangled states could be also applied in medicine and energy transportation.

This paper is organized as follows. In Sec. 2, we constructed the mathematical expressions of the packaged entangled states of a particle-antiparticle pair and discussed their properties. In Sec. 3, we discussed the possible applications of these new entangled states. First, we discussed how to teleport a particle/antiparticle to a place at a distance using the packaged entangled states. In this process, the classical channel is removed due to the particle-antiparticle annihilation phenomenon. Second, we discussed how to transfer a packaged entangled state from two particles to two other particles. Finally, we showed that the collapse of packaged entangled states contributes to the origin of matter-antimatter asymmetry (or baryogenesis) of the observable universe. In Sec. 4, we compared the difference between the packaged entangled states and the entangled states studied in early literatures.

\section{Theory}

In this section, we will use charge conjugation to construct the mathematical expression of the packaged entangled states of a particle-antiparticle pair. As mentioned before, the packaged entangled states encapsulate all the necessary physical quantities for completely identifying the particles. Furthermore, the packaged entangled states should be the eigenstates of some operator. In this sense, one can design possible experimental methods to test the existence of the packaged entangled states in the future.

Let us first denote the particle quantum state as $|P\rangle$ and antiparticle quantum state as $|\bar{P}\rangle$. It is known that $|P\rangle$ and $|\bar{P}\rangle$ are symmetrical in the sense of charge, i.e., the charge of $|P\rangle$ and $|\bar{P}\rangle$ are equal in quantity but with opposite signs. From particle physics $^{35,36,39}$ we know that $|P\rangle$ and $|\bar{P}\rangle$ can be interchanged by the charge conjugation operator $C$, i.e., $C|P\rangle=|\bar{P}\rangle$ and $C|\bar{P}\rangle=|P\rangle$. This mutual transformation 
indicates that $|P\rangle$ and $|\bar{P}\rangle$ could be superimposed in the sense of wave function under certain conditions.

It is well known that the charge conjugation operator $C$ not only reverses the sign of the particle's electric charge $(Q)$, but also reverses the sign of all other internal quantum numbers, ${ }^{35}$ i.e., baryon number $(B)$, lepton number $(L)$, isospin $\left(I_{3}\right)$, charm $(C)$, strangeness $(S)$, topness $(T)$, and bottomness $\left(B^{\prime}\right)$. However, the operator $C$ does not change the particles' mass, energy, momentum, and spin. ${ }^{35}$ In this sense, we may explicitly define the notations $|P\rangle$ and $|\bar{P}\rangle$ in the present paper as

$$
\begin{aligned}
& |P\rangle=\left|Q, B, L, I_{3}, C, S, T, B^{\prime}\right\rangle, \\
& |\bar{P}\rangle=\left|-Q,-B,-L,-I_{3},-C,-S,-T,-B^{\prime}\right\rangle .
\end{aligned}
$$

The early literatures ${ }^{40,41}$ have discussed the superposition states with different charges. In fact, the superposition states with different charges exist in the systems like well-studied bound "particle-antiparticle" pairs $|P \bar{P}\rangle$, which are the eigenstates of $C$. ${ }^{\text {b }}$ Recently, the experiments in condensed matter physics ${ }^{42-45}$ have confirmed the existence of Majorana fermion, i.e., the superposition state of electron-hole pairs created by operators $\gamma_{j}=c_{j}^{\dagger}+c_{j}$, where $c_{j}^{\dagger}$ is the creation operators of electrons or annihilation operators of holes, and $c_{j}$ is the annihilation operators of electrons or creation operators of holes. The entanglement in these bound states are related to spin and orbital angular momentum. Here, we will extend these ideas to the packaged entangled states that encapsulate physical quantities $Q, B, L, I_{3}, C, S, T$, and $B^{\prime}$.

\subsection{Packaged entangled states with C-symmetry}

We first wish to construct the packaged entangled states with $C$-symmetry. Let us now consider the following separable states of a particle-antiparticle pair $A$ and $B$, i.e.,

$$
\begin{aligned}
\left|\Theta^{+}\right\rangle_{A B} & =|P\rangle_{A}|\bar{P}\rangle_{B}, \\
\left|\Theta^{-}\right\rangle_{A B} & =|\bar{P}\rangle_{A}|P\rangle_{B},
\end{aligned}
$$

and their linear combinations (superpositions),

$$
\begin{aligned}
\left|\Psi^{+}\right\rangle_{A B} & =\frac{1}{\sqrt{2}}\left(|P\rangle_{A}|\bar{P}\rangle_{B}+|\bar{P}\rangle_{A}|P\rangle_{B}\right), \\
\left|\Psi^{-}\right\rangle_{A B} & =\frac{1}{\sqrt{2}}\left(|P\rangle_{A}|\bar{P}\rangle_{B}-|\bar{P}\rangle_{A}|P\rangle_{B}\right) .
\end{aligned}
$$

\footnotetext{
${ }^{\mathrm{b}}$ Not all particle (antiparticle) states, but only neutral systems (with zero total charge) are the eigenstates of the charge conjugation operator $C,{ }^{35}$ such as $\gamma, \pi^{0}$, and a bound "particle-antiparticle" pair $|P \bar{P}\rangle$ etc. The bound pair $|P \bar{P}\rangle$ is an eigenstate of the charge conjugation operator $C$ because it satisfies the relation $^{35} C(|P \bar{P}\rangle)=(-1)^{J}|P \bar{P}\rangle$, where $J=L+S$ is the total angular momentum quantum number, $L$ is the orbital angular momentum quantum number, and $S$ is the total spin quantum number. For bosons $|b\rangle, S$ is an even number and the relation reduces to $C(|b\rangle|\bar{b}\rangle)=(-1)^{L}|b\rangle|\bar{b}\rangle$.
} 
Apply the charge conjugation operator $C$ to the separable states, we have $C\left|\Theta^{+}\right\rangle_{A B}=\left|\Theta^{-}\right\rangle_{A B}$ and $C\left|\Theta^{-}\right\rangle_{A B}=\left|\Theta^{+}\right\rangle_{A B}$. This shows that the separable states $\left|\Theta^{ \pm}\right\rangle_{A B}$ are not the eigenstates of $C$.

Let us now apply the operator $C$ to the superposition states $\left|\Psi^{ \pm}\right\rangle_{A B}$, we have,

$$
\begin{aligned}
C\left|\Psi^{ \pm}\right\rangle_{A B} & =\frac{1}{\sqrt{2}}\left[C\left(|P\rangle_{A}|\bar{P}\rangle_{B}\right) \pm C\left(|\bar{P}\rangle_{A}|P\rangle_{B}\right)\right], \\
& =\frac{1}{\sqrt{2}}\left(|\bar{P}\rangle_{A}|P\rangle_{B} \pm|P\rangle_{A}|\bar{P}\rangle_{B}\right), \\
& = \pm\left|\Psi^{ \pm}\right\rangle_{A B} .
\end{aligned}
$$

Equation (2.4) shows that the superposition states $\left|\Psi^{ \pm}\right\rangle_{A B}$ are the eigenstates of the charge conjugation operator $C$. On the other hand, one can easily show that $C=C^{\dagger}{ }^{46}$ This means that $C$ is a Hermitian operator and is therefore an observable physical quantity. As the eigenstates of $C$, therefore, $\left|\Psi^{ \pm}\right\rangle_{A B}$ must exist.

The separable states $\left|\Theta^{ \pm}\right\rangle_{A B}$ have a fundamental character: each of their particles is either a particle or an antiparticle, the particles' identities are determinate. But the superposition states $\left|\Psi^{ \pm}\right\rangle_{A B}$ (eigenstates of $C$ ) are entangled states because they cannot be expressed as the direct product of the particle state and antiparticle state. ${ }^{1,2}$ A fundamental character of $\left|\Psi^{ \pm}\right\rangle_{A B}$ is that each of their particles is a superposition of a particle and an antiparticle, i.e., it is partially a particle and partially an antiparticle. Therefore, one cannot tell which one is a particle and which one is an antiparticle. When performing a measurement on $A$, it will collapse into either a particle $|P\rangle_{A}$, or an antiparticle $|\bar{P}\rangle_{A}$. If $A$ collapse into a particle $|P\rangle_{A}$, then $B$ will collapse into an antiparticle $|\bar{P}\rangle_{B}$, i.e., $\left|\Psi^{ \pm}\right\rangle_{A B} \rightarrow|P\rangle_{A}|\bar{P}\rangle_{B}$. If $A$ collapse into an antiparticle $|\bar{P}\rangle_{A}$, then $B$ will collapse into a particle $|P\rangle_{B}$, i.e., $\left|\Psi^{ \pm}\right\rangle_{A B} \rightarrow \pm|\bar{P}\rangle_{A}|P\rangle_{B}$

Finally, let us explicitly write out the packaged entangled states $\left|\Psi^{ \pm}\right\rangle_{A B}$ (see Eq. (2.3)) using the quantum numbers $\left(Q, B, L, I_{3}, C, S, T, B^{\prime}\right)$, which are packaged together by the charge conjugation operator $C$. The quantum numbers $\left(Q, B, L, I_{3}, C, S, T, B^{\prime}\right)$ should be entangled together in $\left|\Psi^{ \pm}\right\rangle_{A B}$. Therefore, $\left|\Psi^{ \pm}\right\rangle_{A B}$ cannot be written as the product of the sub quantum states related to the individual quantum numbers (freedoms). Referring to Eq. (2.1), we may rewrite Eq. (2.3) as

$$
\begin{aligned}
\left|\Psi^{ \pm}\right\rangle_{A B}= & \frac{1}{\sqrt{2}}\left(\left|Q, B, L, I_{3}, C, S, T, B^{\prime}\right\rangle_{A}\left|-Q,-B,-L,-I_{3},-C,-S,-T,-B^{\prime}\right\rangle_{B}\right. \\
& \left. \pm\left|-Q,-B,-L,-I_{3},-C,-S,-T,-B^{\prime}\right\rangle_{A}\left|Q, B, L, I_{3}, C, S, T, B^{\prime}\right\rangle_{B}\right) .
\end{aligned}
$$

Equation (2.5) explicitly shows that the quantum numbers $\left(Q, B, L, I_{3}, C\right.$, $\left.S, T, B^{\prime}\right)$ are packaged as an entirety in the packaged entangled states $\left|\Psi^{ \pm}\right\rangle_{A B}$. This occurs due to the charge conjugation operator $C$. Thus, the quantum numbers cannot be added in or taken out separately from $\left|\Psi^{ \pm}\right\rangle_{A B}$. Furthermore, a particle and an antiparticle are different types of particles. It means that $\left|\Psi^{ \pm}\right\rangle_{A B}$ package 
different particles as an entirety. Due to these reasons, we call the quantum states $\left|\Psi^{ \pm}\right\rangle_{A B}$ as packaged entangled states.

\subsection{Packaged entangled states with C-symmetry breaking}

The packaged entangled states $\left|\Psi^{ \pm}\right\rangle_{A B}$ in Eq. (2.3) are constructed on the basis of a particle-antiparticle pair in which the total charge is conserved (zero). They strictly obey the law of charge conservation in the wave function collapse. From a mathematical point of view, however, there should be other forms of packaged entangled states in which the total charge are not conserved in the wave function collapse, i.e., the total charge before the wave function collapse is not equal to that after the wave function collapse. Let us now construct the mathematical expressions for these new packaged entangled states.

Consider the following two quantum states of a particle pair,

$$
\begin{aligned}
\left|\Phi^{+}\right\rangle_{A B} & =\frac{1}{\sqrt{2}}\left(|P\rangle_{A}|P\rangle_{B}+|\bar{P}\rangle_{A}|\bar{P}\rangle_{B}\right), \\
\left|\Phi^{-}\right\rangle_{A B} & =\frac{1}{\sqrt{2}}\left(|P\rangle_{A}|P\rangle_{B}-|\bar{P}\rangle_{A}|\bar{P}\rangle_{B}\right) .
\end{aligned}
$$

Applying the charge conjugation operator $C$ to $\left|\Phi^{ \pm}\right\rangle_{A B}$, we have

$$
\begin{aligned}
C\left|\Phi^{ \pm}\right\rangle_{A B} & =\frac{1}{\sqrt{2}}\left[C\left(|P\rangle_{A}|P\rangle_{B}\right) \pm C\left(|\bar{P}\rangle_{A}|\bar{P}\rangle_{B}\right)\right], \\
& =\frac{1}{\sqrt{2}}\left[|\bar{P}\rangle_{A}|\bar{P}\rangle_{B} \pm|P\rangle_{A}|P\rangle_{B}\right], \\
& = \pm\left|\Phi^{ \pm}\right\rangle_{A B} .
\end{aligned}
$$

Equation (2.7) shows that $\left|\Phi^{ \pm}\right\rangle_{A B}$ are also the eigenstates of charge conjugation operator $C$. Because $C$ is a Hermitian operator (or an observable physical quantity), ${ }^{\mathrm{c}}$ its eigenstates $\left|\Phi^{ \pm}\right\rangle_{A B}$ must exist.

Similar to $\left|\Psi^{ \pm}\right\rangle_{A B}$, the states $\left|\Phi^{ \pm}\right\rangle_{A B}$ are also entangled states because they cannot be expressed as the direct product of the particle states and antiparticle states. ${ }^{1,2}$ Furthermore, as the eigenstates of the charge conjugation operator $C$, the entangled states $\left|\Phi^{ \pm}\right\rangle_{A B}$ also package in all the physical properties capable of completely identifying the particles, i.e., the particle's electric charge $(Q)$, baryon number $(B)$, lepton number $(L)$, isospin $\left(I_{3}\right)$, charm $(C)$, strangeness $(S)$, topness $(T)$, and bottomness $\left(B^{\prime}\right)$.

The packaged entangled states $\left|\Phi^{ \pm}\right\rangle_{A B}$ have an interesting property. If a measurement is performed on the particle pair, $\left|\Phi^{+}\right\rangle_{A B}\left(\right.$ or $\left.\left|\Phi^{-}\right\rangle_{A B}\right)$ will collapse and break the $C$-symmetry (the symmetry of physical laws under the charge conjugation operator $C) .{ }^{35,36,39}$ More specifically, if a measurement is performed on $A$, it will

${ }^{\mathrm{c}}$ Apply the charge conjugation operator $C$ to the particle state $|P\rangle$ twice, we have $C^{2}|P\rangle=C|\bar{P}\rangle=|P\rangle$. This gives $C^{2}=1$. On the other hand, the normalization condition $\left\langle P\left|C^{\dagger} C\right| P\right\rangle=\langle\bar{P} \mid \bar{P}\rangle=1$ gives $C^{\dagger} C=1$. Thus, we have $C=C^{\dagger}$. 
collapse into either a particle $|P\rangle_{A}$, or an antiparticle $|\bar{P}\rangle_{A}$. If $A$ collapses into a particle $|P\rangle_{A}$, then $B$ will also collapse into a particle $|P\rangle_{B}$, i.e., $\left|\Phi^{ \pm}\right\rangle_{A B} \rightarrow|P\rangle_{A}|P\rangle_{B}$. If $A$ collapses into an antiparticle $|\bar{P}\rangle_{A}$, then $B$ will also collapse into an antiparticle $|\bar{P}\rangle_{B}$, i.e., $\left|\Phi^{ \pm}\right\rangle_{A B} \rightarrow \pm|\bar{P}\rangle_{A}|\bar{P}\rangle_{B}$. This process breaks the $C$-symmetry of the particle-antiparticle pair. Therefore, the law of charge conservation does not hold in this process.

\subsection{Generalization to $M(>2)$-particle systems}

The packaged entangled states constructed in Eqs. (2.3) and (2.6) are valid for 2-particle systems only. We will now generalize them to $M>2$-particle systems.

The quantum states of the $M(>2)$-particle system can be classified using the number of antiparticles included in the state. Let us first consider the separable states of this system and denote them by $|\Theta\rangle_{i j}$, where the suffix $i$ represents the number of antiparticles and $j$ labels the $j$ th combination of $i$ antiparticles in total $M$ particles. The value of $j$ can be $j=1, \ldots, Q$, where $Q=\left(\begin{array}{c}M \\ i\end{array}\right)=\frac{M(M-1) \cdots(M-i+1)}{i !}$ is the number of $i$-combinations from $M$ elements. Thus, we have

$$
\begin{aligned}
& |\Theta\rangle_{i 1}=|\bar{P}\rangle_{1}|\bar{P}\rangle_{2} \cdots|\bar{P}\rangle_{i-1}|\bar{P}\rangle_{i}|P\rangle_{i+1}|P\rangle_{i+2} \cdots|P\rangle_{M-1}|P\rangle_{M}, \\
& |\Theta\rangle_{i 2}=|\bar{P}\rangle_{1}|\bar{P}\rangle_{2} \cdots|\bar{P}\rangle_{i-1}|P\rangle_{i}|\bar{P}\rangle_{i+1}|P\rangle_{i+2} \cdots|P\rangle_{M-1}|P\rangle_{M}, \\
& \cdots \cdots
\end{aligned}
$$

In fact, the separable states in Eq. (2.8) can be obtained by doing various permutations to the first one. One can see that these separable states are not symmetrical. We can form the symmetrical state by adding the separable states (all possible permutations), i.e.,

$$
|\Theta\rangle_{i}=\frac{1}{\sqrt{Q}}\left(|\Theta\rangle_{i 1}+|\Theta\rangle_{i 2}+|\Theta\rangle_{i 3}+\cdots+|\Theta\rangle_{i Q}\right) .
$$

Equation (2.9) shows that $|\Theta\rangle_{i}$ is an entangled state, but it is not the eigenstate of charge conjugation operator $C$. Finally, we can obtain the eigenstate of operator $C$ by doing a linear combinations on $|\Theta\rangle_{i}$ and its charge conjugation state $|\bar{\Theta}\rangle_{i}=C|\Theta\rangle_{i}$, i.e.,

$$
\begin{aligned}
\left|\Phi^{ \pm}\right\rangle_{i} & =\frac{1}{\sqrt{2}}\left(|\Theta\rangle_{i} \pm|\bar{\Theta}\rangle_{i}\right) \\
& =\frac{1}{\sqrt{2 Q}}\left[\left(|\Theta\rangle_{i 1}+|\Theta\rangle_{i 2} \cdots+|\Theta\rangle_{i Q}\right) \pm\left(|\bar{\Theta}\rangle_{i 1}+|\bar{\Theta}\rangle_{i 2}+\cdots+|\bar{\Theta}\rangle_{i Q}\right)\right]
\end{aligned}
$$

One can show that $C\left|\Phi^{ \pm}\right\rangle_{i}=\frac{1}{\sqrt{2}}\left(|\bar{\Theta}\rangle_{i} \pm|\Theta\rangle_{i}\right)= \pm\left|\Phi^{ \pm}\right\rangle_{i}$. This means that the packaged entangled states $\left|\Phi^{ \pm}\right\rangle_{i}$ are the eigenstates of charge conjugation operator $C$.

It should be mentioned that, due to the particle-antiparticle symmetry, the separable states have following symmetry (using the charge conjugation operator $C$ ),

$$
|\bar{\Theta}\rangle_{i j}=C|\Theta\rangle_{i j}=|\Theta\rangle_{(M-i) j},
$$


where the formula $\left(\begin{array}{c}M \\ i\end{array}\right)=\left(\begin{array}{c}M \\ M-i\end{array}\right)$ is used. ${ }^{46}$ Using Eq. (2.11), one can easily write out the separable states of the systems with more than $\left[\frac{M}{2}\right]$ (here $\left[\frac{M}{2}\right]$ denotes the integer part of $\frac{M}{2}$ ) antiparticles (i.e., $M-i$ antiparticles) as follows:

$$
\begin{aligned}
& |\bar{\Theta}\rangle_{01}=|\Theta\rangle_{M 1}, \\
& |\bar{\Theta}\rangle_{1 j}=|\Theta\rangle_{(M-1) j}, \\
& |\bar{\Theta}\rangle_{2 j}=|\Theta\rangle_{(M-2) j}, \\
& \cdots \cdots,
\end{aligned}
$$

Let us now explicitly write out some special cases as follows:

(i) Zero antiparticle $(i=0)$.

The total number of such separable states is $\left(\begin{array}{c}M \\ 0\end{array}\right)=1$.

$$
|\Theta\rangle_{01}=|P\rangle_{1}|P\rangle_{2}|P\rangle_{3} \cdots|P\rangle_{M-1}|P\rangle_{M}
$$

The packaged entangled states are (see Eq. $(2.10))^{47}$

$$
\left|\Phi^{ \pm}\right\rangle_{0}=\frac{1}{\sqrt{2}}\left(|\Theta\rangle_{01} \pm|\bar{\Theta}\rangle_{01}\right)
$$

If $M=2$, then Eq. (2.13) reduces to Eq. (2.6).

(ii) One antiparticle $(i=1)$.

The total number of such separable states is $\left(\begin{array}{c}M \\ 1\end{array}\right)=M$.

$$
\begin{aligned}
&|\Theta\rangle_{11}=|\bar{P}\rangle_{1}|P\rangle_{2}|P\rangle_{3} \cdots|P\rangle_{M-1}|P\rangle_{M} \\
&|\Theta\rangle_{12}=|P\rangle_{1}|\bar{P}\rangle_{2}|P\rangle_{3} \cdots|P\rangle_{M-1}|P\rangle_{M} \\
& \cdots \cdots \\
&|\Theta\rangle_{1 M}=|P\rangle_{1}|P\rangle_{2}|P\rangle_{3} \cdots|P\rangle_{M-1}|\bar{P}\rangle_{M}
\end{aligned}
$$

The symmetrical state is

$$
|\Theta\rangle_{1}=\frac{1}{\sqrt{M}}\left(|\Theta\rangle_{11}+|\Theta\rangle_{12}+|\Theta\rangle_{13}+\cdots+|\Theta\rangle_{1 M}\right)
$$

and packaged entangled states are

$$
\left|\Phi^{ \pm}\right\rangle_{1}=\frac{1}{\sqrt{2}}\left(|\Theta\rangle_{1} \pm|\bar{\Theta}\rangle_{1}\right)
$$

If $M=2$, then Eq. (2.15) reduces to Eq. (2.3). 
(iii) Two antiparticles $(i=2)$.

The total number of such separable states is $B=\left(\begin{array}{c}M \\ 2\end{array}\right)=\frac{M(M-1)}{2 !}$.

$$
\begin{gathered}
|\Theta\rangle_{21}=|\bar{P}\rangle_{1}|\bar{P}\rangle_{2}|P\rangle_{3} \cdots|P\rangle_{M-1}|P\rangle_{M}, \\
|\Theta\rangle_{22}=|\bar{P}\rangle_{1}|P\rangle_{2}|\bar{P}\rangle_{3} \cdots|P\rangle_{M-1}|P\rangle_{M}, \\
\cdots \cdots .
\end{gathered}
$$

The symmetrical state is

$$
|\Theta\rangle_{2}=\frac{1}{\sqrt{B}}\left(|\Theta\rangle_{21}+|\Theta\rangle_{22}+|\Theta\rangle_{23}+\cdots+|\Theta\rangle_{2 B}\right)
$$

and packaged entangled states are

$$
\left|\Phi^{ \pm}\right\rangle_{2}=\frac{1}{\sqrt{2}}\left(|\Theta\rangle_{2} \pm|\bar{\Theta}\rangle_{2}\right) .
$$

(iv) $\left[\frac{M}{2}\right]$ antiparticles $\left(i=\left[\frac{M}{2}\right]\right)$.

Let $\left[\frac{M}{2}\right]$ denote the integer part of $\frac{M}{2}$. If $M$ is an even number, then $\left[\frac{M}{2}\right]=\frac{M}{2}$. If $M$ is an odd number, then $C=\frac{M}{2}-1<\left[\frac{M}{2}\right]<\frac{M}{2}$. The total number of such separable states is $\left(\begin{array}{c}M \\ {[M / 2]}\end{array}\right)=\frac{M(M-1) \cdots(M-[M / 2]+1)}{[M / 2] !}$.

$$
\begin{aligned}
|\Theta\rangle_{\left[\frac{M}{2}\right] 1} & =|\bar{P}\rangle_{1}|\bar{P}\rangle_{2} \cdots|\bar{P}\rangle_{\left[\frac{M}{2}\right]-1}|\bar{P}\rangle_{\left[\frac{M}{2}\right]}|P\rangle_{\left[\frac{M}{2}\right]+1}|P\rangle_{\left[\frac{M}{2}\right]+2} \cdots|P\rangle_{M-1}|P\rangle_{M}, \\
|\Theta\rangle_{\left[\frac{M}{2}\right] 2} & =|\bar{P}\rangle_{1}|\bar{P}\rangle_{2} \cdots|\bar{P}\rangle_{\left[\frac{M}{2}\right]-1}|P\rangle_{\left[\frac{M}{2}\right]}|\bar{P}\rangle_{\left[\frac{M}{2}\right]+1}|P\rangle_{\left[\frac{M}{2}\right]+2} \cdots|P\rangle_{M-1}|P\rangle_{M},
\end{aligned}
$$

The symmetrical state is

$$
|\Theta\rangle_{\left[\frac{M}{2}\right]}=\frac{1}{\sqrt{C}}\left(|\Theta\rangle_{\left[\frac{M}{2}\right] 1}+|\Theta\rangle_{\left[\frac{M}{2}\right] 2}+\cdots+|\Theta\rangle_{\left[\frac{M}{2}\right] C}\right)
$$

and packaged entangled states are

$$
\left|\Phi^{ \pm}\right\rangle_{\left[\frac{M}{2}\right]}=\frac{1}{\sqrt{2}}\left(|\Theta\rangle_{\left[\frac{M}{2}\right]} \pm|\bar{\Theta}\rangle_{\left[\frac{M}{2}\right]}\right) .
$$

From the above discussions, we see that the packaged entangled states $\left|\Phi^{ \pm}\right\rangle_{i}$ have the following properties (Let $N_{P}$ be the number of $P_{\mathrm{S}}$ and $N_{\bar{P}}$ be the number of $\bar{P}$ s in $\left.|\Theta\rangle_{i}\right)$ :

(1) The collapse of the packaged entangled states $\left|\Phi^{ \pm}\right\rangle_{i}$ will reduce them to one of the separable states $|\Theta\rangle_{i j}$ (see Eq. (2.8)) or $\pm|\bar{\Theta}\rangle_{i j}$, but not the entangled state $|\Theta\rangle_{i}$ (see Eq. (2.9)) or $\pm|\bar{\Theta}\rangle_{i}$.

(2) If $M$ (the number of total particles in the system) is an even number and $N_{P}=N_{\bar{P}}$, then the $C$-symmetry holds in the collapse of these wave functions. For example, if a measurement is performed on $\left|\Phi^{ \pm}\right\rangle_{\left[\frac{M}{2}\right]}$ (see Eq. (2.19)), then they 
will either collapse into one of the separable states $|\Theta\rangle_{\left[\frac{M}{2}\right] j}$ or $\pm|\bar{\Theta}\rangle_{\left[\frac{M}{2}\right] j}$. The $C$-symmetry holds in these processes.

(3) If $M$ is an even number but $N_{P} \neq N_{\bar{P}}$, then the $C$-symmetry does not hold in the collapse of $\left|\Phi^{ \pm}\right\rangle_{i}$. For example, if a measurement is performed on $\left|\Phi^{ \pm}\right\rangle_{0}$ (see Eq. (2.13)), then they will either collapse into the separable states $|\Theta\rangle_{01}$ or $\pm|\bar{\Theta}\rangle_{01}$. These processes break the $C$-symmetry. Therefore, the $C$-symmetry does not hold in the collapse of these wave functions.

(4) If $M$ is an odd number, then we always have $N_{P} \neq N_{\bar{P}}$. Therefore, all the packaged entangled states have $C$-symmetry breaking.

\subsection{Properties and verification scheme}

\subsubsection{Annihilation of particles in packaged entangled states}

It is well known that a particle and an antiparticle will annihilate each other when they encounter. ${ }^{48}$ Thus, one may ask what will happen when an external particle (from a particle source) encounters a particle in a packaged entangled state? For simplicity, let us now choose the packaged entangled state of two particles $\left|\Psi^{ \pm}\right\rangle_{A B}$ (see Eq. (2.3)) and $\left|\Phi^{ \pm}\right\rangle_{A B}$ (see Eq. (2.6)) to carry out the analysis.

Recall that external perturbation or a measurement on the particles in a packaged entangled state will cause the wave function to collapse. Because each particle in the packaged entangled state is a superposition of a particle and an antiparticle, the collapse of the packaged entangled state is then not random, but has a partiality depending on the external particle due to the particle-antiparticle annihilation phenomenon. In other words, when an external particle $X$ (from a particle source) encounters a particle $A$ that is in a packaged entangled state, the particle-antiparticle annihilation phenomenon ${ }^{\mathrm{d} 49}$ will force $A$ to collapse into a particle conjugating to $X$ (with every internal quantum number of $A$ opposite to that of $X$ ), or project $A$ onto a state conjugating to that of $X$. Thereafter, $X$ and $A$ annihilate each other. This is not a process of continuous evolution via the Schrödinger equation. ${ }^{50}$ More specifically,

(1) If $X$ is a particle (denoted as $|P\rangle_{X}$ ), then $A$ will collapse into an antiparticle $|\bar{P}\rangle_{A}$. The collapse of total wave function may be expressed as

$$
\begin{aligned}
|P\rangle_{X}\left|\Psi^{ \pm}\right\rangle_{A B} & \rightarrow \pm\left(|P\rangle_{X}|\bar{P}\rangle_{A}\right)|P\rangle_{B}, \\
|P\rangle_{X}\left|\Phi^{ \pm}\right\rangle_{A B} & \rightarrow \pm\left(|P\rangle_{X}|\bar{P}\rangle_{A}\right)|\bar{P}\rangle_{B} .
\end{aligned}
$$

(2) If $X$ is an antiparticle (denoted as $|\bar{P}\rangle_{X}$ ), then $A$ will collapse into a particle $|P\rangle_{A}$. The collapse of total wave function may be expressed as

$$
\begin{aligned}
|\bar{P}\rangle_{X}\left|\Psi^{ \pm}\right\rangle_{A B} & \rightarrow\left(|\bar{P}\rangle_{X}|P\rangle_{A}\right)|\bar{P}\rangle_{B}, \\
|\bar{P}\rangle_{X}\left|\Phi^{ \pm}\right\rangle_{A B} & \rightarrow\left(|\bar{P}\rangle_{X}|P\rangle_{A}\right)|P\rangle_{B} .
\end{aligned}
$$

\footnotetext{
d When a low-energy elementary particle-antiparticle pair annihilates, say an electron annihilates with a positron, they can only produce two or more photons. But when a composite particle-antiparticle pair annihilates, say a proton annihilates with an antiproton, they will produce multiple particles, such as photons, electrons, positrons, and neutrinos.
} 
One can see that each particle in the packaged entangled states can annihilate with both a particle and an antiparticle. This property could be used for testing the existence of packaged entangled states, particle-antiparticle teleportation, and transfer of packaged entangled states.

\subsubsection{Entanglement measurement}

We now wish to investigate the tools for quantifying the packaged entanglement and the possible methods for detecting them. This might provide more direct physical ideas and methods to confirm the existence of packaged entangled states.

(1) Correlation measurement. From the above discussion, we know that the particles in a packaged entangled state are not independent, but correlated. Therefore, when performing a measurement on one of the particles, the result of other one is not arbitrary, but has a fixed relationship with the former. Let function $M(i)$ denote the measured result of the $i$ th particle, i.e., $M(i)=+1$ for a particle and $M(i)=-1$ for an antiparticle. The correlation of particles $A$ and $B$ is then defined as the expectation value, the product of the measurements, $E(A, B)=\langle M(A) M(B)\rangle$.

Consider a particle-antiparticle pair that is created in packaged entangled state

$$
\left|\Psi^{+}\right\rangle_{A B}=\frac{1}{\sqrt{2}}\left(|P\rangle_{A}|\bar{P}\rangle_{B}+|\bar{P}\rangle_{A}|P\rangle_{B}\right)
$$

one of the particles (particle $A$ ) is sent to Alice and the other (particle $B$ ) is sent to Bob. Because these two particles are superpositions of a particle and an antiparticle, Alice (or Bob) can control their particles to be either a particle or an antiparticle (see Sec. 2.4.1). Suppose Alice makes a measurement on particle $A$. If $A$ is found to be a particle $|P\rangle_{A}(M(A)=+1)$, then $B$ is necessarily an antiparticle $|\bar{P}\rangle_{B}(M(B)=-1)$. The corresponding correlation is $E(A, B)=-1$. On the contrary, if $A$ is found to be an antiparticle $|\bar{P}\rangle_{A}(M(A)=-1)$, then $B$ is necessarily a particle $|P\rangle_{B}$ $(M(B)=+1)$. The corresponding correlation is $E(A, B)=-1$.

Similarly, Alice and Bob can repeat the measurements using other packaged entangled states. The results are summarized in Table 1.

Table 1. Correlation measurements.

\begin{tabular}{lccc}
\hline Packaged entangled states & Alice's result & Bob's result & Correlation $E(A, B)$ \\
\hline$\left|\Psi^{+}\right\rangle_{A B}$ & $|P\rangle_{A}$ & $|\bar{P}\rangle_{B}$ & -1 \\
& $|\bar{P}\rangle_{A}$ & $|P\rangle_{B}$ & -1 \\
$\left|\Psi^{-}\right\rangle_{A B}$ & $|P\rangle_{A}$ & $|\bar{P}\rangle_{B}$ & -1 \\
& $|\bar{P}\rangle_{A}$ & $-|P\rangle_{B}$ & +1 \\
$\left|\Phi^{+}\right\rangle_{A B}$ & $|P\rangle_{A}$ & $|P\rangle_{B}$ & +1 \\
& $|\bar{P}\rangle_{A}$ & $|\bar{P}\rangle_{B}$ & +1 \\
$\left|\Phi^{-}\right\rangle_{A B}$ & $|P\rangle_{A}$ & $|P\rangle_{B}$ & +1 \\
& $|\bar{P}\rangle_{A}$ & $-|\bar{P}\rangle_{B}$ & -1 \\
\hline
\end{tabular}


(2) von Neumann entanglement entropy. von Neumann entanglement entropy ${ }^{51}$ is a tool for quantifying entanglement. It is usually defined as (for bi-particle states) where $\rho_{A B}$ is the density matrix:

$$
S\left(\rho_{A B}\right)=-\operatorname{Tr}\left(\rho_{A B} \log _{2} \rho_{A B}\right) .
$$

If $\lambda_{i}$ are the eigenvalues of the density matrix $\rho_{A B}$, then Eq. (2.20) can be rewritten as

$$
S\left(\rho_{A B}\right)=-\sum_{i} \lambda_{i} \log _{2} \lambda_{i}
$$

Let us now choose packaged entangled state $\left|\Psi^{+}\right\rangle_{A B}=\frac{1}{\sqrt{2}}\left(|P\rangle_{A}|\bar{P}\rangle_{B}+|\bar{P}\rangle_{A}|P\rangle_{B}\right)$ (see Eq. (2.3a)) to carry out the calculation. The density matrix $\rho_{A B}$ is

$$
\begin{aligned}
\rho_{A B}= & \left|\Psi^{+}\right\rangle_{A B}\left\langle\left.\Psi^{+}\right|_{A B}\right. \\
= & \frac{1}{2}\left(| P \rangle _ { A } | \overline { P } \rangle _ { B } \langle P | _ { A } \langle \overline { P } | _ { B } + | P \rangle _ { A } | \overline { P } \rangle _ { B } \left\langle\overline { P } | _ { A } \left\langle\left.P\right|_{B}\right.\right.\right. \\
& +|\bar{P}\rangle_{A}|P\rangle_{B}\left\langle\left. P\right|_{A}\left\langle\left.\bar{P}\right|_{B}+\mid \bar{P}\right\rangle_{A} \mid P\right\rangle_{B}\left\langle\left.\bar{P}\right|_{A}\left\langle\left. P\right|_{B}\right) .\right.
\end{aligned}
$$

Using matrix notations, one can rewrite Eq. (2.22) as

$$
\rho_{A B}=\frac{1}{2}\left(\begin{array}{llll}
0 & 0 & 0 & 0 \\
0 & 1 & 1 & 0 \\
0 & 1 & 1 & 0 \\
0 & 0 & 0 & 0
\end{array}\right) .
$$

The four eigenvalues of Eq. (2.23) are $\left\{\lambda_{1}, \lambda_{2}, \lambda_{3}, \lambda_{4}\right\}=\{1,0,0,0\}$. Substituting these eigenvalues into Eq. (2.21), we have $S\left(\rho_{A B}\right)=0$.

Similarly, one can repeat the above calculation using packaged entangled states $\left|\Psi^{-}\right\rangle_{A B},\left|\Phi^{+}\right\rangle_{A B}$, and $\left|\Phi^{-}\right\rangle_{A B}$. The results show that the von Neumann entanglement entropies of all these packaged entangled states are zero. This is consistent with the theory.

\subsubsection{Possible verification scheme}

Based on the above discussion, we may use the following experimental scheme to test whether a particle-antiparticle pair is in a packaged entangled state or a separable state.

Suppose that Alice has invented an experimental method for creating particleantiparticle pairs in the packaged entangled states $\left|\Psi^{ \pm}\right\rangle_{A B}$. Now Alice uses this method to create a particle-antiparticle pair and then send one of the particles (particle $A$ ) to annihilate with an external similar particle $X$ (see Fig. 1). Repeating this procedure for a number of times, Alice then starts to calculate the particle annihilating rate.

(a) If the particle-antiparticle pairs are in one of the packaged entangled states $\left|\Psi^{ \pm}\right\rangle_{A B}$, then the annihilation rate would be 1 . This is because each particle in the 


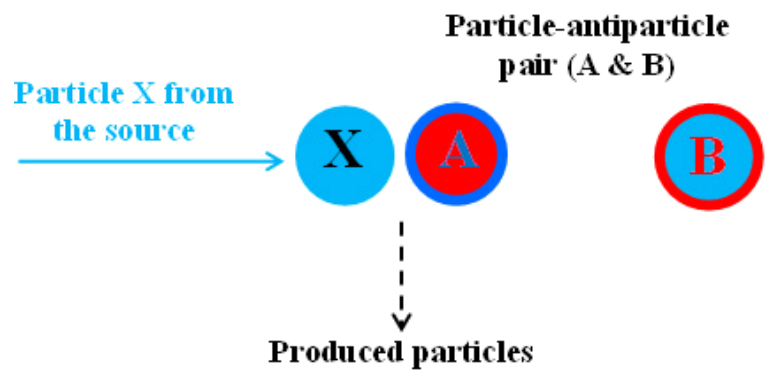

Fig. 1. Schematic diagram for testing the existence of packaged entangled states of particle-antiparticle pairs. If the annihilation rate is 1 , then the pairs are in packaged entangled states. If the annihilation rate is 0.5 , then the pairs are in separable states.

pairs are indeterminate and they can certainly annihilate with the external particles $X$ (it does not matter whether $X$ are particles or antiparticles).

(b) If the particle-antiparticle pairs are in one of the separable states $\left|\Theta^{ \pm}\right\rangle_{A B}$, then the annihilation rate would be 0.5 . This is because each particle in the pairs are determinate and the probability for $A$ to be particles or antiparticles is 0.5 , respectively. Thus, only half of the particles $A$ can annihilate with the external particles $X$.

Finally, Alice can tell whether the particle-antiparticle pairs are in the packaged entangled states $\left|\Psi^{ \pm}\right\rangle_{A B}$ by checking the annihilation rate.

\section{Applications}

\subsection{Particle-antiparticle teleportation with packaged entangled states}

We shall now discuss a possible particle-antiparticle teleportation protocol using the packaged entangled states (see Fig. 2). Here, the particle-antiparticle teleportation

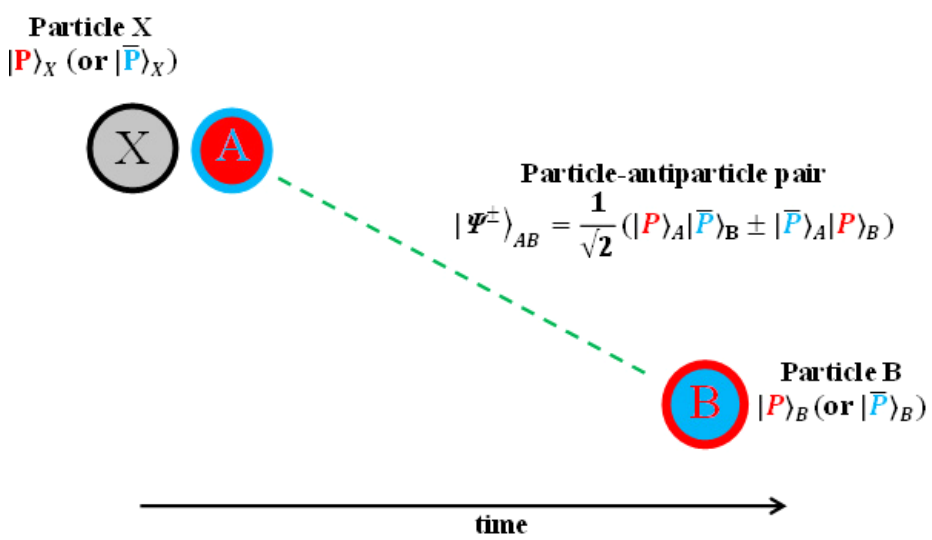

Fig. 2. Schematic diagram for particle-antiparticle teleportation using the packaged entangled states of a particle-antiparticle pair, $\left|\Psi^{ \pm}\right\rangle_{A B}=\frac{1}{\sqrt{2}}\left(|P\rangle_{A}|\bar{P}\rangle_{B} \pm|\bar{P}\rangle_{A}|P\rangle_{B}\right)$, and particle-antiparticle annihilation phenomenon. 
does not mean to transmit a particle to a receiver (from Alice to Bob), but only transmit the packaged quantum information carried by the particle to the receiver. ${ }^{37}$ In the teleportation process, we need to use particle-antiparticle annihilation phenomenon which can be efficiently described by second quantization formalism. But this is not the main point of the teleportation protocol. Our main point is about quantum entanglement and wave function collapse which are related to first quantization.

Let us choose the packaged entangled state $\left|\Psi^{+}\right\rangle_{A B}$ in Eq. (2.3a) to carry out the calculation. One will see that the sender can control the receiver's particle to be a particle (or an antiparticle) in the teleportation process. For the convenience of discussion, the particle-antiparticle teleportation protocol is divided into five steps.

(1) Encoding. To carry out a particle-antiparticle teleportation, Alice needs to encode her information in the form of quantum states of particles and antiparticles. Consider that Alice has a particle $X$ (or a sequence of particles) want to teleport to Bob. $X$ is either a particle $|P\rangle_{X}$ or an antiparticle $|\bar{P}\rangle_{X}$. Without losing generality, let us write out $X$ 's quantum state in a single formula as

$$
|\phi\rangle_{X}=\alpha|P\rangle_{X}+\beta|\bar{P}\rangle_{X},
$$

by assuming that, if $X$ is a particle, then $\alpha=1$ and $\beta=0$; if $X$ is an antiparticle, then $\alpha=0$ and $\beta=1$.

Generally, a single particle is either a particle or an antiparticle (say an electron or a positron). Thus, the quantum information should be a particle-antiparticle sequence as $\ldots,|P\rangle, \ldots,|\bar{P}\rangle, \ldots$

(2) Quantum channel creation. Alice needs a quantum channel, a particle-antiparticle pair in the packaged entangled states, to send the information stored on particle $X$ to Bob. According to the agreement between Alice and Bob, they choose $\left|\Psi^{+}\right\rangle_{A B}=\frac{1}{\sqrt{2}}\left(|P\rangle_{A}|\bar{P}\rangle_{B}+|\bar{P}\rangle_{A}|P\rangle_{B}\right.$ ) (see Eq. (2.3a)) to carry out the teleportation.

After the particle-antiparticle pair is created in $\left|\Psi^{+}\right\rangle_{A B}$, one of them (particle $A$ ) is sent to Alice and the other one (particle $B$ ) is sent to Bob. Before Alice carries out any further operation, the complete state of the three particles $(X, A, B)$ is

$$
\begin{aligned}
|\phi\rangle_{X}\left|\Psi^{+}\right\rangle_{A B}= & \frac{\alpha}{\sqrt{2}}\left(|P\rangle_{X}|P\rangle_{A}|\bar{P}\rangle_{B}+|P\rangle_{X}|\bar{P}\rangle_{A}|P\rangle_{B}\right) \\
& +\frac{\beta}{\sqrt{2}}\left(|\bar{P}\rangle_{X}|P\rangle_{A}|\bar{P}\rangle_{B}+|\bar{P}\rangle_{X}|\bar{P}\rangle_{A}|P\rangle_{B}\right)
\end{aligned}
$$

(3) Sending. Alice can send out her information stored on particle $X$ by annihilating particle $X$ with particle $A$. Referring to Sec. 2.4.1, we know that, when particle $X$ encounters particle $A$, the particle-antiparticle annihilation phenomenon (see footnote $\mathrm{d})^{49}$ will force $A$ to collapse into a particle conjugating to $X$, or project $A$ onto the state $|\bar{\phi}\rangle_{A}=\bar{\alpha}|\bar{P}\rangle_{A}+\bar{\beta}|P\rangle_{A}$ (see Eq. (3.1)). Thereafter, $X$ and $A$ annihilate each other. Meanwhile, the three particle state $|\phi\rangle_{X}\left|\Psi^{+}\right\rangle_{A B}$ (see Eq. (3.2)) should collapse into a final state $\left|\Psi^{+}\right\rangle_{X A B}^{\prime}$ that only has terms including $|P\rangle_{X}|\bar{P}\rangle_{A}$ and 
$|\bar{P}\rangle_{X}|P\rangle_{A}$ (particle-antiparticle annihilation). One can see that only the second term and third term in Eq. (3.2) satisfy these conditions. Therefore, we obtain the final state,

$$
\begin{aligned}
\left|\Psi^{+}\right\rangle_{X A B}^{\prime} & =\alpha\left(|P\rangle_{X}|\bar{P}\rangle_{A}\right)|P\rangle_{B}+\beta\left(|\bar{P}\rangle_{X}|P\rangle_{A}\right)|\bar{P}\rangle_{B} \\
& =\alpha|P \bar{P}\rangle_{X A}|P\rangle_{B}+\beta|\bar{P} P\rangle_{X A}|\bar{P}\rangle_{B} .
\end{aligned}
$$

where $|P \bar{P}\rangle_{X A}$ and $|\bar{P} P\rangle_{X A}$ are the particles produced by the $|P\rangle_{X}|\bar{P}\rangle_{A}$ and $|\bar{P}\rangle_{X}|P\rangle_{A}$ annihilation (see footnote d), respectively.

The reduction $|\phi\rangle_{X}\left|\Psi^{+}\right\rangle_{A B} \rightarrow\left|\Psi^{+}\right\rangle_{X A B}^{\prime}$ indicates that the state of particle $B$ will be modulated after Alice annihilated particle $X$ and $A$. Thus, Alice successfully sents out her information (state of particle $X$ ) to Bob (state of particle $B$ ).

(4) Receiving. Bob can receive the packaged information by measuring the state of particle $B$. Recall that before Alice annihilate particle $X$ with $A$, Bob's particle $B$ was in the packaged entangled state $\left|\Psi^{+}\right\rangle_{A B}$ and was unrelated to particle $X$. After Alice annihilated particle $X$ and $A$, however, particle $B$ collapsed into a separable state identical to that of particle $X$. This can be seen by by referring to Eq. (3.3). Thus, the teleportation process is a simple correspondence between $X$ and $B$, i.e.,

$$
\left(\alpha|P\rangle_{X}+\beta|\bar{P}\rangle_{X}\right) \sim \alpha|P \bar{P}\rangle_{X A}|P\rangle_{B}+\beta|\bar{P} P\rangle_{X A}|\bar{P}\rangle_{B}
$$

This shows that Bob's particle $B$ becomes identical to $X$ after Alice sents out her information by annihilating $X$ with $A$. Therefore, Bob can receive the packaged information of particle $X$ by measuring the state of particle $B$.

(5) Decoding. Referring to Eqs. (3.1) and (3.3), Bob can decode the packaged information sent to him by Alice (carried by particle $X$ ). More specifically, if $X$ is a particle, i.e., $|\phi\rangle_{X}=|P\rangle_{X}$ (see Eq. (3.1)), then Eq. (3.3) becomes $\left|\Psi^{+}\right\rangle_{X A B}^{\prime}=$ $|P \bar{P}\rangle_{X A}|P\rangle_{B}$ and $B$ becomes a particle identical to $X$; If $X$ is an antiparticle, i.e., $|\phi\rangle_{X}=|\bar{P}\rangle_{X}$, then Eq. (3.3) becomes $\left|\Psi^{+}\right\rangle_{X A B}^{\prime}=|\bar{P} P\rangle_{X A}|\bar{P}\rangle_{B}$ and $B$ becomes an antiparticle identical to $X$. The exact correspondence between $X$ and $B$ can be written as: $|P\rangle_{X} \sim|P\rangle_{B},|\bar{P}\rangle_{X} \sim|\bar{P}\rangle_{B}$.

Thus, if Bob measure $B$ and find it is a particle, then he can infer that $X$ is a particle. However, if Bob measures $B$ and finds it is an antiparticle, then he can infer that $X$ is an antiparticle. In this sense, Bob can successfully decode the packaged information sent to him by Alice (carried by $X$ ).

Similarly, one can repeat the above particle teleportation process using the other packaged entangled states:

(1) If one chooses $\left|\Psi^{-}\right\rangle_{A B}$ (see Eq. (2.3b)), then Eq. (3.3) becomes

$$
\left|\Psi^{-}\right\rangle_{X A B}^{\prime}=-\alpha|P \bar{P}\rangle_{X A}|P\rangle_{B}+\beta|\bar{P} P\rangle_{X A}|\bar{P}\rangle_{B}
$$

If $|\phi\rangle_{X}=|P\rangle_{X}$, then $\left|\Psi^{-}\right\rangle_{X A B}^{\prime}=-|P \bar{P}\rangle_{X A}|P\rangle_{B}$. If $|\phi\rangle_{X}=|\bar{P}\rangle_{X}$, then $\left|\Psi^{-}\right\rangle_{X A B}^{\prime}=$ $|\bar{P} P\rangle_{X A}|\bar{P}\rangle_{B}$ 
(2) If one chooses $\left|\Phi^{+}\right\rangle_{A B}$ (see Eq. (2.6)), then Eq. (3.3) becomes

$$
\left|\Phi^{+}\right\rangle_{X A B}^{\prime}=\alpha|P \bar{P}\rangle_{X A}|\bar{P}\rangle_{B}+\beta|\bar{P} P\rangle_{X A}|P\rangle_{B}
$$

If $|\phi\rangle_{X}=|P\rangle_{X}$, then $\left|\Phi^{+}\right\rangle_{X A B}^{\prime}=|P \bar{P}\rangle_{X A}|\bar{P}\rangle_{B}$ ( $B$ becomes an antiparticle conjugating to $X)$. If $|\phi\rangle_{X}=|\bar{P}\rangle_{X}$, then $\left|\Phi^{+}\right\rangle_{X A B}^{\prime}=|\bar{P} P\rangle_{X A}|P\rangle_{B}$ (B becomes an particle conjugating to $X)$.

(3) If one chooses $\left|\Phi^{-}\right\rangle_{A B}$ (see Eq. (2.6)), then Eq. (3.3) becomes

$$
\left|\Phi^{-}\right\rangle_{X A B}^{\prime}=-\alpha|P \bar{P}\rangle_{X A}|\bar{P}\rangle_{B}+\beta|\bar{P} P\rangle_{X A}|P\rangle_{B}
$$

If $|\phi\rangle_{X}=|P\rangle_{X}$, then $\left|\Phi^{-}\right\rangle_{X A B}^{\prime}=-|P \bar{P}\rangle_{X A}|\bar{P}\rangle_{B}$. If $|\phi\rangle_{X}=|\bar{P}\rangle_{X}$, then $\left|\Phi^{-}\right\rangle_{X A B}^{\prime}=$ $|\bar{P} P\rangle_{X A}|P\rangle_{B}$

The above discussion shows that, if Alice and Bob choose $\left|\Psi^{ \pm}\right\rangle_{A B}$ (packaged entangled states with $C$-symmetry, see Eq. (2.3), then Bob can obtain a particle (particle $B$ ) identical to that of Alice (particle $X$ ). However, if Alice and Bob choose $\left|\Phi^{ \pm}\right\rangle_{A B}$ (packaged entangled states with $C$-symmetry breaking, see Eq. (2.6)), then Bob can obtain a particle conjugating to that of Alice.

Using the states $\left|\Psi^{ \pm}\right\rangle_{A B}$, the particle-antiparticle teleportation process satisfies a number of conservation principles, i.e., charge (Q) conservation, baryon number $(B)$ conservation, lepton number $(L)$ conservation, isospin $\left(I_{3}\right)$ conservation, charm $(C)$ conservation, strangeness $(S)$ conservation, topness $(T)$ conservation, and bottomness $\left(B^{\prime}\right)$ conservation. Using the states $\left|\Phi^{ \pm}\right\rangle_{A B}$, however, the particle-antiparticle teleportation process does not satisfy the above conservation principles. But in both cases, the particle-antiparticle teleportation processes satisfy the principles of linear momentum conservation, total energy conservation, and angular momentum conservation.

Finally, let us discuss how to teleport particles to multiple receivers ${ }^{52,53}$ using one of the packaged entangled states in Eq. (2.15), i.e.,

$$
\left|\Phi^{+}\right\rangle_{1}=\frac{1}{\sqrt{2 M}}\left[\left(|\Theta\rangle_{11}+|\Theta\rangle_{12}+\cdots+|\Theta\rangle_{1 M}\right)+\left(|\bar{\Theta}\rangle_{11}+|\bar{\Theta}\rangle_{12}+\cdots+|\bar{\Theta}\rangle_{1 M}\right)\right] .
$$

Consider that Alice has a particle $X$ as described by Eq. (3.1) and wants to teleport it to the $M-1$ receivers: Bob, Carl, David, Edward, Frank, ... . First, send particle 1 in the packaged entangled states $\left|\Phi^{+}\right\rangle_{1}$ to Alice and send the other $M-1$ particles to the multiple receivers, respectively. Before Alice carries out any further operation, the complete state of the $1+M$ particles (see Eq. (3.2)) is

$$
\begin{aligned}
|\phi\rangle_{X}\left|\Phi^{+}\right\rangle_{1}= & \frac{\alpha}{\sqrt{2 M}}|P\rangle_{X}\left[\left(|\Theta\rangle_{11}+|\Theta\rangle_{12}+\cdots+|\Theta\rangle_{1 M}\right)\right. \\
& \left.+\left(|\bar{\Theta}\rangle_{11}+|\bar{\Theta}\rangle_{12}+\cdots+|\bar{\Theta}\rangle_{1 M}\right)\right] \\
& +\frac{\beta}{\sqrt{2 M}}|\bar{P}\rangle_{X}\left[\left(|\Theta\rangle_{11}+|\Theta\rangle_{12}+\cdots+|\Theta\rangle_{1 M}\right)\right. \\
& \left.+\left(|\bar{\Theta}\rangle_{11}+|\bar{\Theta}\rangle_{12}+\cdots+|\bar{\Theta}\rangle_{1 M}\right)\right]
\end{aligned}
$$


Thereafter, Alice sends out her information stored on particle $X$ by annihilating particle $X$ with particle 1 . The $1+M$ particle state $|\phi\rangle_{X}\left|\Phi^{+}\right\rangle_{1}$ (see Eq. (3.8)) should collapse into a final state $\left|\Phi^{+}\right\rangle_{X 1(M-1)}^{\prime}$ that only has terms including $|P\rangle_{X}|\bar{P}\rangle_{1}$ and $|\bar{P}\rangle_{X}|P\rangle_{1}$ (particle-antiparticle annihilation). One can see that only the terms $|\Theta\rangle_{11}$ and $|\bar{\Theta}\rangle_{11}$ in Eq. (3.8) satisfy these conditions. Therefore, we obtain the final state,

$$
\begin{aligned}
\left|\Phi^{+}\right\rangle_{X 1(M-1)}^{\prime}= & \alpha|P\rangle_{X}|\Theta\rangle_{11}+\beta|\bar{P}\rangle_{X}|\bar{\Theta}\rangle_{11} \\
= & \alpha|P\rangle_{X}|\bar{P}\rangle_{1}|P\rangle_{2}|P\rangle_{3} \cdots|P\rangle_{M-1}|P\rangle_{M} \\
& +\beta|\bar{P}\rangle_{X}|P\rangle_{1}|\bar{P}\rangle_{2}|\bar{P}\rangle_{3} \cdots|\bar{P}\rangle_{M-1}|\bar{P}\rangle_{M} \\
= & \alpha|P \bar{P}\rangle_{X 1}|P\rangle_{2}|P\rangle_{3} \cdots|P\rangle_{M-1}|P\rangle_{M} \\
& +\beta|\bar{P} P\rangle_{X 1}|\bar{P}\rangle_{2}|\bar{P}\rangle_{3} \cdots|\bar{P}\rangle_{M-1}|\bar{P}\rangle_{M}
\end{aligned}
$$

If $|\phi\rangle_{X}=|P\rangle_{X}$, then $\left|\Phi^{+}\right\rangle_{X 1(M-1)}^{\prime}=|P \bar{P}\rangle_{X 1}|P\rangle_{2}|P\rangle_{3} \cdots|P\rangle_{M-1}|P\rangle_{M}$. If $|\phi\rangle_{X}=$ $|\bar{P}\rangle_{X}$, then $\left|\Phi^{+}\right\rangle_{X 1(M-1)}^{\prime}=|\bar{P} P\rangle_{X 1}|\bar{P}\rangle_{2}|\bar{P}\rangle_{3} \cdots|\bar{P}\rangle_{M-1}|\bar{P}\rangle_{M}$.

Equation (3.9) shows that, using $\left|\Phi^{+}\right\rangle_{1}$, each receiver can receive a particle identical to particle $X$. This confirms that Alice can teleport particles to multiple receivers.

\subsection{Transfer of packaged entangled states}

As mentioned before, Alice needs a quantum channel (a particle pair in a packaged entangled state) to perform particle-antiparticle teleportation. However, if two unentangled particles are spatially separated by large distance, then it is difficult to put these two particles into a packaged entangled state. In this case, one should consider the possibility of transferring the packaged entangled state from the entangled particles to the objective particles which are originally unrelated.

The purpose of this section is to study the entanglement transfer. The procedure is similar but not equal to the entanglement swapping. ${ }^{54,55}$ The fundamental difference is that the entanglement swapping process uses Bell measurements to swap the entanglements, but here we will use particle-antiparticle annihilation phenomenon to transfer the packaged entanglements. First, let us choose $\left|\Psi^{+}\right\rangle_{A B}=$ $\frac{1}{\sqrt{2}}\left(|P\rangle_{A}|\bar{P}\rangle_{B}-|\bar{P}\rangle_{A}|P\rangle_{B}\right.$ ) (see Eq. (2.3a)) to carry out the calculation (see Fig. 3).

Consider that particles $A$ and $B$ are originally in the packaged entangled state $\left|\Psi^{+}\right\rangle_{A B}$, and particles $C$ and $D$ are in the packaged entangled state $\left|\Psi^{+}\right\rangle_{C D}$, i.e.,

$$
\begin{aligned}
\left|\Psi^{+}\right\rangle_{A B} & =\frac{1}{\sqrt{2}}\left(|P\rangle_{A}|\bar{P}\rangle_{B}+|\bar{P}\rangle_{A}|P\rangle_{B}\right), \\
\left|\Psi^{+}\right\rangle_{C D} & =\frac{1}{\sqrt{2}}\left(|P\rangle_{C}|\bar{P}\rangle_{D}+|\bar{P}\rangle_{C}|P\rangle_{D}\right) .
\end{aligned}
$$

Apparently, $A$ and $D$ are unrelated, $B$ and $C$ are unrelated. Now we wish to connect $A$ and $D$ in a packaged entangled state without touching them. This can be achieved by annihilating $B$ and $C$. 


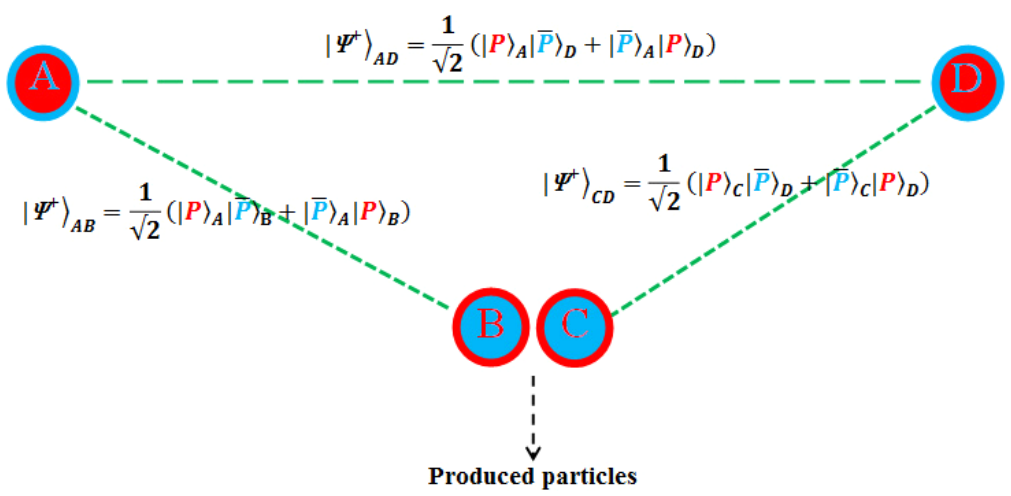

Fig. 3. Schematic diagram for the entanglement transfer from the packaged entangled states $\left|\Psi^{+}\right\rangle_{A B}=$ $\frac{1}{\sqrt{2}}\left(|P\rangle_{A}|\bar{P}\rangle_{B}+|\bar{P}\rangle_{A}|P\rangle_{B}\right)$ and $\left|\Psi^{+}\right\rangle_{C D}=\frac{1}{\sqrt{2}}\left(|P\rangle_{C}|\bar{P}\rangle_{D}+|\bar{P}\rangle_{C}|P\rangle_{D}\right)$ to the packaged entangled state $\left|\Psi^{+}\right\rangle_{A D}=\frac{1}{\sqrt{2}}\left(|P\rangle_{A}|\bar{P}\rangle_{D}+|\bar{P}\rangle_{A}|P\rangle_{D}\right)$

Before Alice carries out any further operation, the complete state of the four particles $(A, B, C, D)$ is

$$
\begin{aligned}
\left|\Psi^{+}\right\rangle_{A B C D}= & \left|\Psi^{+}\right\rangle_{A B}\left|\Psi^{+}\right\rangle_{C D} \\
= & \frac{1}{2}\left(|P\rangle_{A}|\bar{P}\rangle_{B}|P\rangle_{C}|\bar{P}\rangle_{D}+|P\rangle_{A}|\bar{P}\rangle_{B}|\bar{P}\rangle_{C}|P\rangle_{D}\right. \\
& \left.+|\bar{P}\rangle_{A}|P\rangle_{B}|P\rangle_{C}|\bar{P}\rangle_{D}+|\bar{P}\rangle_{A}|P\rangle_{B}|\bar{P}\rangle_{C}|P\rangle_{D}\right)
\end{aligned}
$$

Each particle in the packaged entangled states is a superposition of a particle and an antiparticle. When particle $B$ encounters $C$, the particle-antiparticle annihilation phenomenon (see footnote d) ${ }^{49}$ will force $B$ and $C$ to collapse into a pair of conjugated particles $\left(|P\rangle_{B}|\bar{P}\rangle_{C}\right.$ or $\left.|\bar{P}\rangle_{B}|P\rangle_{C}\right)$. Afterwards, the particle-antiparticle pair $(B, C)$ will annihilate each other. Thus, the $\left|\Psi^{+}\right\rangle_{A B C D}$ in Eq. (3.11) will collapse into a state $\left|\Psi^{++}\right\rangle_{A B C D}^{\prime}$ which only has terms including $|P\rangle_{B}|\bar{P}\rangle_{C}$ and $|\bar{P}\rangle_{B}|P\rangle_{C}$, i.e.,

$$
\begin{aligned}
\left|\Psi^{++}\right\rangle_{A B C D}^{\prime} & =\frac{1}{\sqrt{2}}\left(|P\rangle_{A}|\bar{P}\rangle_{B}|P\rangle_{C}|\bar{P}\rangle_{D}+|\bar{P}\rangle_{A}|P\rangle_{B}|\bar{P}\rangle_{C}|P\rangle_{D}\right) \\
& =\left|\Psi^{+}\right\rangle_{A D}|P \bar{P}\rangle_{B C} .
\end{aligned}
$$

where $\left|\Psi^{+}\right\rangle_{A D}=\frac{1}{\sqrt{2}}\left(|P\rangle_{A}|\bar{P}\rangle_{D}+|\bar{P}\rangle_{A}|P\rangle_{D}\right)$ and $|P \bar{P}\rangle_{B C}$ are the particles produced by the $|P\rangle_{B}|\bar{P}\rangle_{C}$ and $|\bar{P}\rangle_{B}|P\rangle_{C}$ annihilation (see footnote $\mathrm{d}$ ).

Equation (3.12) shows that after the annihilation of particles $B$ and $C$, particles $A$ and $D$ (originally unrelated) is now in the packaged entangled states $\left|\Psi^{+}\right\rangle_{A D}$.

Furthermore, the above transfer process can be performed in a sequence or chain with any number of packaged entanglement pairs, i.e.,

$$
A-\overbrace{B \cdots C}-\overbrace{D \cdots E}-\overbrace{F \cdots G}-\overbrace{H \cdots I}^{H \cdots}-J .
$$

Similarly, one can repeat the transfer process using other combinations of packaged entangled states. The results are summarized in Table 2. 
Table 2. Transfer of packaged entangled states.

\begin{tabular}{lcc}
\hline Combinations in Eq. (3.10) & \multicolumn{1}{c}{ Eq. (3.12) } & Transferred state $|\ldots\rangle_{A D}$ \\
\hline$\left|\Psi^{+}\right\rangle_{A B}\left|\Psi^{+}\right\rangle_{C D}$ & $\left|\Psi^{++}\right\rangle_{A B C D}^{\prime}=\left|\Psi^{+}\right\rangle_{A D}|P \bar{P}\rangle_{B C}$ & $\left|\Psi^{+}\right\rangle_{A D}=\frac{1}{\sqrt{2}}\left(|P\rangle_{A}|\bar{P}\rangle_{D}+|\bar{P}\rangle_{A}|P\rangle_{D}\right)$ \\
$\left|\Psi^{-}\right\rangle_{A B}\left|\Psi^{-}\right\rangle_{C D}$ & $\left|\Psi^{--}\right\rangle_{A B C D}^{\prime}=\left|\Psi^{+}\right\rangle_{A D}|P \bar{P}\rangle_{B C}$ & $\left|\Psi^{+}\right\rangle_{A D}=\frac{1}{\sqrt{2}}\left(|P\rangle_{A}|\bar{P}\rangle_{D}+|\bar{P}\rangle_{A}|P\rangle_{D}\right)$ \\
$\left|\Psi^{+}\right\rangle_{A B}\left|\Psi^{-}\right\rangle_{C D}$ & $\left|\Psi^{+-}\right\rangle_{A B C D}^{\prime}=\left|\Psi^{-}\right\rangle_{A D}|P \bar{P}\rangle_{B C}$ & $\left|\Psi^{-}\right\rangle_{A D}=\frac{1}{\sqrt{2}}\left(|P\rangle_{A}|\bar{P}\rangle_{D}-|\bar{P}\rangle_{A}|P\rangle_{D}\right)$ \\
$\left|\Phi^{+}\right\rangle_{A B}\left|\Phi^{+}\right\rangle_{C D}$ & $\left|\Phi^{++}\right\rangle_{A B C D}^{\prime}=\left|\Psi^{+}\right\rangle_{A D}|P \bar{P}\rangle_{B C}$ & $\left|\Psi^{+}\right\rangle_{A D}=\frac{1}{\sqrt{2}}\left(|P\rangle_{A}|\bar{P}\rangle_{D}+|\bar{P}\rangle_{A}|P\rangle_{D}\right)$ \\
$\left|\Phi^{-}\right\rangle_{A B}\left|\Phi^{-}\right\rangle_{C D}$ & $\left|\Phi^{--}\right\rangle_{A B C D}^{\prime}=-\left|\Psi^{+}\right\rangle_{A D}|P \bar{P}\rangle_{B C}$ & $\left|\Psi^{+}\right\rangle_{A D}=\frac{1}{\sqrt{2}}\left(|P\rangle_{A}|\bar{P}\rangle_{D}+|\bar{P}\rangle_{A}|P\rangle_{D}\right)$ \\
$\left|\Phi^{+}\right\rangle_{A B}\left|\Phi^{-}\right\rangle_{C D}$ & $\left|\Phi^{+-}\right\rangle_{A B C D}^{\prime}=-\left|\Psi^{-}\right\rangle_{A D}|P \bar{P}\rangle_{B C}$ & $\left|\Psi^{-}\right\rangle_{A D}=\frac{1}{\sqrt{2}}\left(|P\rangle_{A}|\bar{P}\rangle_{D}-|\bar{P}\rangle_{A}|P\rangle_{D}\right)$ \\
$\left|\Psi^{+}\right\rangle_{A B}\left|\Phi^{+}\right\rangle_{C D}$ & $\left|\Omega^{++}\right\rangle_{A B C D}^{\prime}=\left|\Phi^{+}\right\rangle_{A D}|P \bar{P}\rangle_{B C}$ & $\left|\Phi^{+}\right\rangle_{A D}=\frac{1}{\sqrt{2}}\left(|P\rangle_{A}|P\rangle_{D}+|\bar{P}\rangle_{A}|\bar{P}\rangle_{D}\right)$ \\
$\left|\Psi^{+}\right\rangle_{A B}\left|\Phi^{-}\right\rangle_{C D}$ & $\left|\Omega^{+-}\right\rangle_{A B C D}^{\prime}=\left|\Phi^{-}\right\rangle_{A D}|P \bar{P}\rangle_{B C}$ & $\left|\Phi^{-}\right\rangle_{A D}=\frac{1}{\sqrt{2}}\left(|P\rangle_{A}|P\rangle_{D}-|\bar{P}\rangle_{A}|\bar{P}\rangle_{D}\right)$ \\
$\left|\Psi^{-}\right\rangle_{A B}\left|\Phi^{+}\right\rangle_{C D}$ & $\left|\Omega^{-+}\right\rangle_{A B C D}^{\prime}=\left|\Phi^{-}\right\rangle_{A D}|P \bar{P}\rangle_{B C}$ & $\left|\Phi^{-}\right\rangle_{A D}=\frac{1}{\sqrt{2}}\left(|P\rangle_{A}|P\rangle_{D}-|\bar{P}\rangle_{A}|\bar{P}\rangle_{D}\right)$ \\
$\left|\Psi^{-}\right\rangle_{A B}\left|\Phi^{-}\right\rangle_{C D}$ & $\left|\Omega^{--}\right\rangle_{A B C D}^{\prime}=\left|\Phi^{+}\right\rangle_{A D}|P \bar{P}\rangle_{B C}$ & $\left|\Phi^{+}\right\rangle_{A D}=\frac{1}{\sqrt{2}}\left(|P\rangle_{A}|P\rangle_{D}+|\bar{P}\rangle_{A}|\bar{P}\rangle_{D}\right)$ \\
\hline
\end{tabular}

The above discussion shows that if one wishes to transfer a $C$-symmetrical packaged entangled states $\left|\Psi^{ \pm}\right\rangle_{A B}$, then he/she needs to choose the identical combinations, i.e., $\left|\Psi^{ \pm}\right\rangle_{A B}\left|\Psi^{ \pm}\right\rangle_{C D}$ and $\left|\Phi^{ \pm}\right\rangle_{A B}\left|\Phi^{ \pm}\right\rangle_{C D}$. However, if one wishes to transfer a $C$-asymmetrical packaged entangled states $\left|\Phi^{ \pm}\right\rangle_{A B}$, then he/she needs to choose the cross combinations, i.e., $\left|\Psi^{ \pm}\right\rangle_{A B}\left|\Phi^{ \pm}\right\rangle_{C D}$.

\subsection{Matter-antimatter asymmetry via packaged entangled states}

Matter-antimatter asymmetry (or baryogenesis) ${ }^{38,56-58}$ usually refers to the imbalance between baryons and antibaryons produced after the Big Bang. The observations have shown that our universe has far more matter than antimatter. ${ }^{59-62}$ This means that our universe either started with more matter than antimatter, or started with equal amount of matter and antimatter but later became matter dominated due to some unknown physical laws. The former seems to be in conflict with relativistic quantum mechanics ${ }^{63}$ and the latter were usually studied by research works. ${ }^{38} \mathrm{Up}$ to now, a number of mechanisms have been proposed to address the matter-antimatter asymmetry, such as Higgs field baryogenesis, ${ }^{64}$ electroweak baryogenesis,${ }^{65,66}$ leptogenesis, ${ }^{67,68}$ Affleck-Dine baryogenesis, ${ }^{69}$ GUT (grand unified theory) baryogenesis, ${ }^{70,71}$ Planck-scale baryogenesis, ${ }^{38}$ etc.

We will now present a new interpretation to the origin of the matter-antimatter asymmetry using the theory of packaged entangled states. Equation (2.6) shows that the total charges of the packaged entangled states $\left|\Phi^{ \pm}\right\rangle_{A B}$ are not conserved in the process of wave function collapse. This phenomenon directly leads to the imbalance between particles and antiparticles, or particle-antiparticle asymmetry. Therefore, the collapse of packaged entangled states with $C$-symmetry breaking may contribute to the matter-antimatter asymmetry of our universe. 
First, we will propose a process of "entanglement selection" to explain why the particles created after the Big Bang are in the packaged entangled states, but not in the separable states. Second, we will show that strong interaction and electromagnetic interaction resulted in a phase transition and therefore leaded to the baryon/ anti-baryon asymmetry. Finally, we will show that the collapse of packaged entangled states with $C$-symmetry breaking satisfies the Sakharov conditions. ${ }^{72}$ Because the collapse of wave function occurred after particle creation, the second quantization formalism is not necessary and first quantization formalism is sufficient to describe the wave function collapse.

\subsubsection{Entanglement selection}

The matter-antimatter asymmetry can be described by the asymmetry parameter ${ }^{73}$ $\eta_{B}=\left(n_{B}-n_{\bar{B}}\right) / n_{\gamma} \simeq n_{B} / n_{\gamma}=(6.19 \pm 0.15) \times 10^{-10}$, where $n_{B}, n_{\bar{B}}$, and $n_{\gamma}$ are the overall number density of baryons, antibaryons, and cosmic background radiation photons, respectively. One can see that $\eta_{B}$ is a very small number. Thus, a mechanism only needs to account for a tiny imbalance produced at a very early time.

(1) Quark creation and annihilation. It is believed that the asymptotically free quarks, gluons, and leptons were created in Quark Epoch (the first millionth of a second after the Big Bang, i.e., $t<10^{-6} \mathrm{~s}$ and $\left.k T>150 \mathrm{MeV}\right)$. An equal amount of quarks and anti-quarks (with zero total charge) should be created according to relativistic quantum mechanics. ${ }^{63}$ This condition is obviously satisfied by both the separable states $\left|\Theta^{ \pm}\right\rangle_{A B}$ in Eq. (2.2), and the packaged entangled states $\left|\Psi^{ \pm}\right\rangle_{A B}$ in Eq. (2.3) and $\left|\Phi^{ \pm}\right\rangle_{A B}$ in Eq. (2.6). Each state that satisfies the particle-antiparticle pair condition is possible to occur according to the principles of quantum mechanics.

If the quarks and anti-quarks were created in the separable states $\left|\Theta^{ \pm}\right\rangle_{A B}$ in Eq. (2.2), then there should be equal amount of quarks and anti-quarks. The quarks would annihilate all the anti-quarks. This is also true for the packaged entangled states $\left|\Psi^{ \pm}\right\rangle_{A B}$ in Eq. (2.3), which have $C$-symmetry and the law of charge conservation holds in the wave function collapse. More specifically, if $A$ collapses into a quark $|P\rangle_{A}$, then $B$ will collapse into an anti-quark $|\bar{P}\rangle_{B}$, i.e., $\left|\Psi^{ \pm}\right\rangle_{A B} \rightarrow|P\rangle_{A}|\bar{P}\rangle_{B}$. If $A$ collapses into an anti-quark $|\bar{P}\rangle_{A}$, then $B$ will collapse into a quark $|P\rangle_{B}$, i.e., $\left|\Psi^{ \pm}\right\rangle_{A B} \rightarrow \pm|\bar{P}\rangle_{A}|P\rangle_{B}$. In other words, an equal amount of quarks and anti-quarks will be produced after the wave function collapses and they will annihilate each other. It should be emphasized that a quark can only annihilate with an anti-quark of the same flavor. Thus, the quarks and anti-quarks created in Eqs. (2.2) and (2.3) will not live long and will go back to photons.

However, if the quarks and anti-quarks were created in the packaged entangled states $\left|\Phi^{ \pm}\right\rangle_{A B}$ in Eq. (2.6), then the collapse of these wave function will break $C$-symmetry. The law of charge conservation does not hold in this process. More specifically, if $A$ collapses into a quark $|P\rangle_{A}$, then $B$ will also collapse into a quark $|P\rangle_{B}$, i.e., $\left|\Phi^{ \pm}\right\rangle_{A B} \rightarrow|P\rangle_{A}|P\rangle_{B}$. If $A$ collapses into an anti-quark $|\bar{P}\rangle_{A}$, then $B$ will also collapse into an anti-quark $|\bar{P}\rangle_{B}$, i.e., $\left|\Phi^{ \pm}\right\rangle_{A B} \rightarrow \pm|\bar{P}\rangle_{A}|\bar{P}\rangle_{B}$. In other words, an 
unequal amount of quarks and anti-quarks were produced after the wave function collapses. Thus, the quarks and anti-quarks created in Eq. (2.6) may survive and live long.

(2) Phase transition. From a collective point of view, however, even if every quark-antiquark pair were created in the packaged entangled states $\left|\Phi^{ \pm}\right\rangle_{A B}$, it is still possible that an equal amount of quarks and anti-quarks will be produced after the collapse of all states $\left|\Phi^{ \pm}\right\rangle_{A B}$. This is because there is an equal probability for each $\left|\Phi^{ \pm}\right\rangle_{A B}$ to collapse into either $|P\rangle_{A}|P\rangle_{B}$ or $\pm|\bar{P}\rangle_{A}|\bar{P}\rangle_{B}$. After the collapse of all these $\left|\Phi^{ \pm}\right\rangle_{A B}$, the total number of quarks will be equal to that of anti-quarks. These quarks and anti-quarks will annihilate each other and go back to photons. Fortunately, this did not happen due to the strong interaction between quarks and gluons which leaded to a phase transition. Let us now prove it.

After the packaged entangled states $\left|\Phi^{ \pm}\right\rangle_{A B}$ collapse into quarks (or antiquarks), the strong interaction between the quarks (or antiquarks) will reduce the quarks' (or antiquarks') energy and combine them into baryons (or antibaryons). Generally, the quark-quark potential due to the strong interaction between quarks can be written as $V_{q q}=-\frac{4}{3} \frac{\alpha_{s}}{r}+k r$, where $\alpha_{s}$ is the quark-gluon coupling, $r$ is the distance between quarks, and $k$ is confinement constant. ${ }^{35,36}$ Because each baryon is made up of three quarks, the total quark-quark potential of each baryon can be approximately written out as $V_{\text {Baryon }} \approx 3\left(-\frac{4}{3} \frac{\alpha_{s}}{r_{A}}+k r_{A}\right)$, where $r_{A}$ is the average distance between quarks.

Recall the definition of baryon number (density) $B=\frac{1}{3}\left(n_{q}-n_{\bar{q}}\right)$, where $n_{q}$ is the number (density) of quarks and $n_{\bar{q}}$ is the number (density) of antiquarks. According to this definition, a baryon has a baryon number of +1 and an antibaryon has a baryon number of -1 . Thus, the total potential of quarks in the baryons (or antibaryons), $V_{t}$, can be written out as,

$$
V_{t} \approx 3\left(-\frac{4}{3} \frac{\alpha_{s}}{r_{A}}+k r_{A}\right)|B|,
$$

where $|B|$ is the absolute value of $B$.

Let us now use symbol $E_{0}$ to denote energy density of all quarks created in the packaged entangled states $\left|\Phi^{ \pm}\right\rangle_{A B}$ (before the collapse of wave function). If the collapse of all packaged entangled states result in an equal amount of quarks and antiquarks, then these quarks and antiquarks will annihilate each other and go back to photons. The total energy will remain in the value of $E_{0}$. However, if all the packaged entangled states collapse into quarks (or antiquarks), then the energy density of quarks (or antiquarks) will reduce to $E_{0}-V_{t}$. Collecting all these terms, the total energy density of quarks can be written out as

$$
E_{t}=\left\{\begin{array}{ll}
E_{0} & \text { before the collapse of the packaged entangled states } \\
E_{0}-V_{t} & \text { after the collapse of the packaged entangled states }
\end{array} .\right.
$$

Equation (3.14) shows that the energy density of all quarks, $E_{t}$, is a decreasing function of the absolute value of baryon number (density) $B$ (see Fig. 4). The packaged entangled states are states with higher energy $\left(E_{0}\right)$. However, the strong 


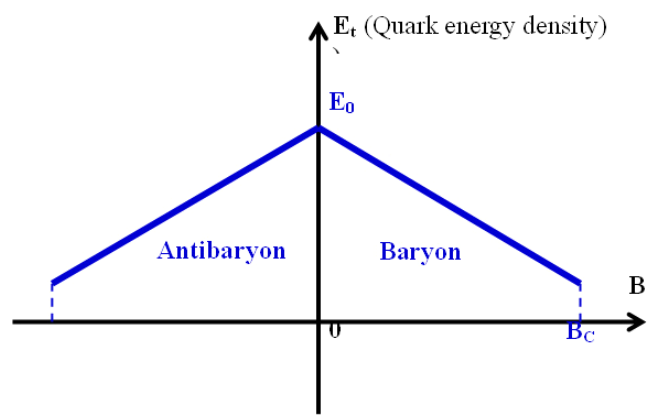

Fig. 4. Schematic diagram for the quark energy density $E_{t}$ as a function of baryon number $B$. An spontaneous symmetry breaking occurs due to the quark-quark potential after the collapse of packaged entangled states. With the expansion of universe, the baryon number stops increasing at the value $\left|B_{C}\right|$ where the total energy density (or temperature) of the universe reduced to the lowest value for creating new quarks/anti-quarks $(150 \mathrm{MeV})$.

interaction between quarks (or antiquarks) made it energy favorable for more packaged entangled states to collapse into baryons (or antibaryons). They experienced a process of spontaneous symmetry breaking and therefore leaded to a phase transition that resulted in matter-antimatter asymmetry.

With the expansion of the universe, the temperature reduces to a critical value where the energy density is not enough to create new quarks/anti-quarks $(k T<150 \mathrm{MeV})$. Finally, part of the baryons will annihilate an equal amount of antibaryons as the universe expanded and cooled down. However, the remnant particles that are not annihilated will survive. This should contribute to the tiny residue asymmetry parameter $\eta_{B} \cdot{ }^{73}$

(3) Leptogenesis. It should be also mentioned that, once the imbalance between baryons and antibaryons is formed, there is a non-zero net charge which will generate an electric field. This field will interact with the leptons/antileptons in packaged entangled states. The interaction is described by the Coulomb potential $V_{\mathrm{em}}=-\frac{\alpha}{r}$. Thus, the collapse of the packaged entangled state of leptons $\left(\left|\Phi^{ \pm}\right\rangle_{A B}\right)$ is not random, but has a partiality depending on the electric field generated by the baryons (or antibaryons). In other words, the electric field baryons (or antibaryons) will cause the leptons/antileptons in the packaged entangled field to collapse into particles with a charge conjugating to that of the baryons (or antibaryons). This means that the baryogenesis results in leptogenesis.

Now, we can summarize the entanglement selection process based on the packaged entangled states with $C$-symmetry breaking: due to particle-antiparticle annihilation, the particles created in the separable states and packaged entangled states with $C$-symmetry are short-lived, but the particles created in the packaged entangled states with $C$-symmetry breaking can be long-lived and survive until today due to phase transition caused by the quark-quark potential. The entanglement selection process is similar to the idea of universal selection from the universal Darwinism (generalized Darwinism). ${ }^{74-76}$ 


\subsubsection{Packaged entangled states and Sakharov conditions}

In 1967, Sakharov ${ }^{72}$ proposed three necessary conditions for a physical process that can lead to the matter-antimatter asymmetry: (1) Violation of baryon number B, (2) Violation of $C$-symmetry and CP-symmetry, (3) Departure from thermal equilibrium. We shall now choose the packaged entangled states $\left|\Phi^{+}\right\rangle_{A B}$ (see Eq. (2.6a)) to show that the collapse of a packaged entangled state with $C$-symmetry breaking satisfies the Sakharov conditions. ${ }^{72}$

(1) Violation of baryon number $B$. All quarks $(u, d, t, b, c, s)$ carry a baryon number $^{35,36} B=1 / 3$ and their anti-quarks carry a baryon number $B=-1 / 3$. Let us now assume that, in the packaged entangled state $\left|\Phi^{+}\right\rangle_{A B}$ (see Eq. (2.6a)), the $|P\rangle_{\mathrm{s}}$ represent quarks and $|\bar{P}\rangle_{\mathrm{s}}$ represent anti-quarks. Therefore, the total baryon number of $\left|\Phi^{+}\right\rangle_{A B}$ is $B=0$. Under the external perturbation, if $\left|\Phi^{+}\right\rangle_{A B}$ collapses into the separable state $|P\rangle_{A}|P\rangle_{B}$, then the variation of baryon number is $\Delta B=2 / 3$. However, if $\left|\Phi^{+}\right\rangle_{A B}$ collapses into the separable state $|\bar{P}\rangle_{A}|\bar{P}\rangle_{B}$, then the variation of baryon number is $\Delta B=-2 / 3$. This means that the total baryon number $B$ is violated in either case during the collapse of wave function.

Similarly, if the $|P\rangle_{\mathrm{S}}$ and $|\bar{P}\rangle_{\mathrm{S}}$ in $\left|\Phi^{+}\right\rangle_{A B}$ represent the leptons and anti-leptons, then the total lepton number $L$ is violated during the collapse of wave function. In fact, the collapse of $\left|\Phi^{+}\right\rangle_{A B}$ also violates the other packaged quantum numbers, i.e., $Q, I_{3}, C, S, T$, and $B^{\prime}$.

(2) Violation of C-symmetry and CP-symmetry. Recall that $\left|\Phi^{+}\right\rangle_{A B}$ is an eigenstate of the charge conjugation operator $C$ (see Eq. (2.7)). Applying $C$ to $\left|\Phi^{+}\right\rangle_{A B}$, we have

$$
C\left|\Phi^{+}\right\rangle_{A B}=\left|\Phi^{+}\right\rangle_{A B}
$$

For the separable states $|P\rangle_{A}|P\rangle_{B}$ and $|\bar{P}\rangle_{A}|\bar{P}\rangle_{B}$, we have

$$
C\left(|P\rangle_{A}|P\rangle_{B}\right)=|\bar{P}\rangle_{A}|\bar{P}\rangle_{B}, \quad \text { and } \quad C\left(|\bar{P}\rangle_{A}|\bar{P}\rangle_{B}\right)=|P\rangle_{A}|P\rangle_{B}
$$

The $C$-symmetry is obviously conserved in Eq. (3.15), but not conserved in Eq. (3.16). This shows that the $C$-symmetry breaks in the process of wave function collapse: $\left|\Phi^{+}\right\rangle_{A B} \rightarrow|P\rangle_{A}|P\rangle_{B}$, or $\left|\Phi^{+}\right\rangle_{A B} \rightarrow|\bar{P}\rangle_{A}|\bar{P}\rangle_{B}$.

The parity operator $P$ reverses the space coordinates, i.e., $P: \mathbf{r} \rightarrow-\mathbf{r}$. Applying $P$ to $\left|\Phi^{+}\right\rangle_{A B}$, we have $P\left|\Phi^{+}\right\rangle_{A B}=p\left|\Phi^{+}\right\rangle_{A B}$, where $p= \pm 1$ is the eigenvalues of $P$. The exact value of $p$ depends on the exchange symmetry of the particle states. Similarly, we have $P\left(|P\rangle_{A}|P\rangle_{B}\right)=p|P\rangle_{A}|P\rangle_{B}$ and $P\left(|\bar{P}\rangle_{A}|\bar{P}\rangle_{B}\right)=p|\bar{P}\rangle_{A}|\bar{P}\rangle_{B}$.

Now applying the product operator CP to $\left|\Phi^{+}\right\rangle_{A B}$, we have

$$
\mathrm{CP}\left|\Phi^{+}\right\rangle_{A B}=p\left|\Phi^{+}\right\rangle_{A B}
$$

Applying CP to the separable states $|P\rangle_{A}|P\rangle_{B}$ and $|\bar{P}\rangle_{A}|\bar{P}\rangle_{B}$, we have

$$
\mathrm{CP}\left(|P\rangle_{A}|P\rangle_{B}\right)=p|\bar{P}\rangle_{A}|\bar{P}\rangle_{B}, \quad \text { and } \quad \mathrm{CP}\left(|\bar{P}\rangle_{A}|\bar{P}\rangle_{B}\right)=p|P\rangle_{A}|P\rangle_{B}
$$


The product CP cannot send $|P\rangle_{A}|P\rangle_{B}$ or $|\bar{P}\rangle_{A}|\bar{P}\rangle_{B}$ back to $\left|\Phi^{+}\right\rangle_{A B}$. Thus, the CP-symmetry ${ }^{39,77}$ breaks in the process of wave function collapse: $\left|\Phi^{+}\right\rangle_{A B} \rightarrow$ $|P\rangle_{A}|P\rangle_{B}$ or $\left|\Phi^{+}\right\rangle_{A B} \rightarrow|\bar{P}\rangle_{A}|\bar{P}\rangle_{B}$

(3) Departure from thermal equilibrium. In fact, the expansion of universe assures the departure from thermal equilibrium. ${ }^{57}$ But we would like to show that the collapse of packaged entangled state with $C$-symmetry breaking further assures the departure from thermal equilibrium.

The particles in packaged entangled state $\left|\Phi^{+}\right\rangle_{A B}$ are indeterminate. Now consider that $\left|\Phi^{+}\right\rangle_{A B}$ collapses into a separable state, i.e., $\left|\Phi^{+}\right\rangle_{A B} \rightarrow|P\rangle_{A}|P\rangle_{B}$ (or $\left.|\bar{P}\rangle_{A}|\bar{P}\rangle_{B}\right)$. However, the particles in the separable state $|P\rangle_{A}|P\rangle_{B}\left(\right.$ or $\left.|\bar{P}\rangle_{A}|\bar{P}\rangle_{B}\right)$ are determinate, i.e., they are either particles or antiparticles. This means that the separable states $|P\rangle_{A}|P\rangle_{B}$ (or $|\bar{P}\rangle_{A}|\bar{P}\rangle_{B}$ ) cannot go back to the packaged entangled state $\left|\Phi^{+}\right\rangle_{A B}$. Therefore, the wave function collapses, $\left|\Phi^{+}\right\rangle_{A B} \rightarrow|P\rangle_{A}|P\rangle_{B}$ (or $\left.|\bar{P}\rangle_{A}|\bar{P}\rangle_{B}\right)$, is an irreversible process. Further, the particles in the separable states are identical particles and they cannot annihilate each other. These show that the collapse of packaged entangled state with $C$-symmetry breaking assures the departure from thermal equilibrium.

\section{Discussion}

We have studied the properties of packaged entangled states and their applications. Let us now further discuss the difference by comparing to other theories.

(1) Characteristics of packaged entangled states. The packaged entangled states are strongly related to the charge conjugation operator $C$ which packages a number of quantum numbers $\left(Q, B, L, I_{3}, C, S, T, B^{\prime}\right)$ as an entirety. These entangled quantum numbers are fixed and cannot be added in or taken out separately. The particles in the packaged entangled states are indeterminate. This means that each particle in the packaged entangled states is partially a particle and partially an antiparticle. In other words, a packaged entangled state is an entangled state of particle and antiparticle, i.e., an entangled state of different particles. Apparently, the hyperentanglement and multimode entangled states do not have these properties. They are the entangled states of several physical quantities of identical particles. These difference result in the difference between the particle-antiparticle teleportation protocol described in this paper and other quantum teleportation protocols described in early literatures, ${ }^{9,53}$ and the difference between the entanglement transfer described in this paper and quantum swapping described in early literatures.

On the other hand, the charge of a particle and an antiparticle are equal in quantity but with opposite signs, i.e., a particle and an antiparticle are symmetrical in the sense of charge. As discussed before, the particles in a packaged entangled state are a superposition of a particle and an antiparticle. Thus, their charges are 
indeterminate. If we refer a particle's charge as its gender, then the particles in the packaged entangled states are hermaphroditic particles. In this sense, the identities of the particles in a packaged entangled states are indeterminate.

(2) Particle-antiparticle teleportation. In early quantum teleportation protocols, ${ }^{9,53}$ Alice sends out the information of particle $X$ by performing a Bell measurement on particles $X$ and $A$. This measurement has four possible results. Thereafter, Alice needs a classical channel to inform Bob about her Bell measurement result. In the present particle-antiparticle teleportation protocol, however, Alice sents out the information of $X$ by annihilating $X$ with $A$. Alice's experimental result is fixed by the particle-antiparticle annihilation phenomenon. Thus, Bob's result has a fixed relationship with that of $X$ and he can decode the information directly. The classical channel between Alice and Bob is then removed, which indicates the possibility of superluminal communication. This is consistent with the recent experimental results which have shown that the speed of "spooky action at a distance" is at least 10,000 times of the speed of light. ${ }^{78}$

In the particle-antiparticle teleportation process, Alice can modulate the receiver's particle (particle $B$ ) to be a particle or an antiparticle at a distance by choosing particle $X$ to be a particle or an antiparticle. Therefore, Alice can control particle $B$ whether to annihilate or not to annihilate with its environment particles at will. This property may be applied in medicine, such as positron emission tomography (PET) ${ }^{79}$ positron annihilation spectroscopy (PAS), and the band structure measurements in solid state physics as well. Furthermore, it may also be applied in remote control. Finally, the particle-antiparticle teleportation process could be used to transport energy because the particle-antiparticle annihilation process can release a large amount of energy.

(3) Transfer of packaged entangled states. In the transfer of packaged entangled states, the outcome is fixed due to the particle-antiparticle annihilation phenomenon. But in quantum swapping, ${ }^{54,55}$ the outcome is not fixed and there are four possible results due to the Bell measurement.

(4) External physical quantities. As mentioned before, the charge conjugation operator $C$ packages a number of internal quantum numbers $\left(Q, B, L, I_{3}, C\right.$, $\left.S, T, B^{\prime}\right)$, but it does not change the particles' mass, energy, momentum, and spin. This means that the packaged entangled states do not include these external physical quantities. The relationship between the internal and external physical quantities will be discussed in a forthcoming paper.

(5) Matter-antimatter asymmetry. The present paper used first quantization formalism (quantum mechanics) to describe the mechanism that caused the matterantimatter asymmetry. The main idea is the collapse of wave function of the packaged entangled states. The matter-antimatter asymmetry was formed after particle creation. However, the other theories ${ }^{64,65,67,69,70}$ used second quantization formalism (quantum field theory) to describe the mechanism that caused the 
matter-antimatter asymmetry. The main ideas are the interactions between elementary particles. The matter-antimatter asymmetry was formed in the process of particle creation.

The entanglement selection process indicates that it is a random result that our universe is made of matter, but not anti-matter. More specifically, it depends on the initial condition at the moment of wave function collapse. For example, if the packaged entangled states $\left|\Phi^{ \pm}\right\rangle_{A B}$ collapse and happened to roll into $|P\rangle_{A}|P\rangle_{B}$, then an universe made of matter is created. However, if $\left|\Phi^{ \pm}\right\rangle_{A B}$ happened to roll into $\pm|\bar{P}\rangle_{A}|\bar{P}\rangle_{B}$, then an anti-universe made of antimatter is created.

Furthermore, the packaged entangled states and entanglement selection process may indicate the possibility of multiverse. ${ }^{80-82}$ This is because the entanglement selection process results in the random origin of the universe, which means that the structure and composition of a universe is not unique. This multiple origin indicates that the existence of universe may be not unique, but multiple.

The matter-antimatter asymmetry via the collapse of packaged entangled states with $C$-symmetry breaking occurred after particle creation. It does not conflict with the mechanisms proposed in early literatures, which occurred in the process of particle creation. All these mechanisms may coexist.

\section{Conclusion}

We have shown that particles and antiparticles can form packaged entangled states. These states can be divided into two types, i.e., $C$-symmetrical and $C$-asymmetrical. The packaged entangled states are the eigenstates of charge conjugation operator. The species of particles in the packaged entangled states are indeterminate. They are superpositions of a particle and an antiparticle. We proposed a protocol for teleporting a particle or an antiparticle to a large distance using the packaged entangled states. Different to early studies, the particle-antiparticle teleportation protocol introduced here does not need a classical channel due to the particle-antiparticle annihilation phenomenon. One can teleport a particle identical to the original particle to the receiver using the packaged entangled states with $C$-symmetry, but teleport a particle conjugating to the original particle to the receiver using the packaged entangled states with $C$-symmetry breaking. One can also transfer a packaged entangled state from a particle pair to another particle pair. Finally, we show that the collapse of packaged entangled states with $C$-symmetry breaking contributes to the matter-antimatter asymmetry of the observed universe. The particles created in separable states and packaged entangled states with $C$-symmetry are short-lived, but the particles created in the packaged entangled states with $C$-symmetry breaking can be long-lived and survive until today. The collapse of packaged entangled states with $C$-symmetry breaking satisfies the Sakharov conditions, i.e., violation of baryon number $\mathrm{B}$, violation of $C$-symmetry and CP-symmetry, and departure from thermal equilibrium. 


\section{References}

1. R. Horodecki, P. Horodecki, M. Horodecki and K. Horodecki, Quantum entanglement, Rev. Mod. Phys. 81 (2009) 865-942, doi: https://doi.org/10.1103/RevModPhys.81.865.

2. H.-K. Lo, S. Popescu and T. Spiller (Eds.), Introduction to Quantum Computation and Information (World Scientific, River-Edge, 1998).

3. M. A. Nielsen and I. L. Chuang, Quantum Computation and Quantum Information (Cambridge University Press, New York, 2010).

4. A. Einstein, B. Podolsky and N. Rosen, Can quantum-mechanical description of physical reality be considered complete? Phys. Rev. 47 (1935) 777, doi: https://doi.org/10.1103/ PhysRev.47.777.

5. J. S. Bell, On the Einstein-Podolsky-Rosen Paradox, Physics 1 (1964) 195-200.

6. J. Bell, Speakable and Unspeakable in Quantum Mechanics (Cambridge University Press, Cambridge, 1987), p. 65.

7. S. J. Freedman and J. F. Clauser, Experimental test of local hidden-variable theories, Phys. Rev. Lett. 28 (1972) 938, doi: https://doi.org/10.1103/PhysRevLett.28.938.

8. A. Aspect, P. Grangier and G. Roger, Experimental realization of Einstein-PodolskyRosen-Bohm Gedankenexperiment: A new violation of Bell's inequalities, Phys. Rev. Lett. 49 (1982) 91, doi: https://doi.org/10.1103/PhysRevLett.49.91.

9. C. H. Bennett, G. Brassard, C. Crepeau, R. Jozsa, A. Peres and W. K. Wootters, Teleporting an unknown quantum state via dual classical and Einstein-Podolsky-Rosen channels, Phys. Rev. Lett. 70 (1993) 1895, doi: https://doi.org/10.1103/PhysRevLett.70.1895.

10. D. Bouwmeester, J.-W. Pan, K. Mattle, M. Eibl, H. Weinfurter and A. Zeilinger, Experimental quantum teleportation, Nature 390 (1997) 575-579, doi: 10.1038/37539.

11. D. Boschi, S. Branca, F. DeMartini, L. Hardy and S. Popescu, Experimental realization of teleporting an unknown pure quantum state via dual classical and Einstein-PodolskyRosen channels, Phys. Rev. Lett. 80 (1998) 1121-1125, doi: https://doi.org/10.1103/ PhysRevLett.80.1121.

12. M. N. Leuenberger, M. E. Flatte and D. D. Awschalom, Teleportation of electronic manyqubit states encoded in the electron spin of quantum dots via single photons, Phys. Rev. Lett. 94 (2005) 107401, doi: https://doi.org/10.1103/PhysRevLett.94.107401.

13. W. Pfaff, B. J. Hensen, H. Bernien, S. B. van Dam, M. S. Blok, T. H. Taminiau, M. J. Tiggelman, R. N. Schouten, M. Markham, D. J. Twitchen and R. Hanson, Unconditional quantum teleportation between distant solid-state quantum bits, Science 345 (2014) 532, doi: $10.1126 /$ science.1253512.

14. H. Krauter, D. Salart, C. A. Muschik, J. M. Petersen, H. Shen, T. Fernholz and E. S. Polzik, Deterministic quantum teleportation between distant atomic objects, Nat. Phys. 9 (2013) 400-404, doi: 10.1038/nphys2631.

15. J. Hofmann, M. Krug, N. Ortegel, L. Gerard, M. Weber, W. Rosenfeld and H. Weinfurter, Heralded entanglement between widely separated atoms, Science 337 (2012) 72-75, doi: $10.1126 /$ science. 1221856 .

16. M. Riebe, H. Haffner, C. F. Roos, W. Hänsel, J. Benhelm, G. P. T. Lancaster, T. W. Karber, C. Becher, F. Schmidt-Kaler, D. F. V. James and R. Blatt, Deterministic quantum teleportation with atoms, Nature 429 (2004) 734-737, doi: 10.1038/ nature02570.

17. M. D. Barrett, J. Chiaverini, T. Schaetz, J. Britton, W. M. Itano, J. D. Jost, E. Knill, C. Langer, D. Leibfried, R. Ozeri and D. J. Wineland, Deterministic quantum teleportation of atomic qubits, Nature 429 (2004) 737-739, doi: 10.1038/nature02608. 
18. S. Olmschenk, D. N. Matsukevich, P. Maunz, D. Hayes, L.-M. Duan and C. Monroe, Quantum teleportation between distant matter qubits, Science 323 (2009) 486-489, doi: 10.1126/science.1167209.

19. Y. Nakamura, Yu. A. Pashkin and J. S. Tsai, Coherent control of macroscopic quantum states in a single-Cooper-pair box, Nature 398 (1999) 786-788, doi: 10.1038/19718.

20. M. Baur, A. Fedorov, L. Steffen, S. Filipp, M. P. da Silva and A. Wallraff, Benchmarking a quantum teleportation protocol in superconducting circuits using tomography and an entanglement witness, Phys. Rev. Lett. 108 (2012) 040502, doi: https://doi.org/10.1103/ PhysRevLett.108.040502.

21. P. G. Kwiat, Hyper-entangled states, J. Mod. Opt. 44 (1997) 2173, doi: 10.1080/ 09500349708231877.

22. J. T. Barreiro, N. K. Langford, N. A. Peters and P. G. Kwiat, Generation of hyperentangled photon pairs, Phys. Rev. Lett. 95 (2005) 260501, doi: https://doi.org/10.1103/ PhysRevLett.95.260501.

23. J. Chen, J. Fan, M. D. Eisaman and A. Migdall, Generation of high-flux hyperentangled photon pairs using a microstructure-fiber Sagnac interferometer, Phys. Rev. A 77 (2008) 053812, doi: https://doi.org/10.1103/PhysRevA.77.053812.

24. G. Vallone, R. Ceccarelli, F. De Martini and P. Mataloni, Hyperentanglement of two photons in three degrees of freedom, Phys. Rev. A 79 (2009) 030301(R), doi: https://doi. org/10.1103/PhysRevA.79.030301.

25. K. Liu, J. Guo, C. Cai, S. Guo and J. Gao, Experimental generation of continuousvariable hyperentanglement in an optical parametric oscillator, Phys. Rev. Lett. 113 (2014) 170501, doi: https://doi.org/10.1103/PhysRevLett.113.170501.

26. A. Gatti, R. Zambrini, M. San Miguel and L. A. Lugiato, Multiphoton multimode polarization entanglement in parametric down-conversion, Phys. Rev. A 68 (2003) 053807, doi: https://doi.org/10.1103/PhysRevA.68.053807.

27. V. Giovannetti, D. Frustaglia and F. Taddei, Electronic Hong-Ou-Mandel interferometer for multimode entanglement detection, Phys. Rev. B 74 (2006) 115315, doi: https://doi. org/10.1103/PhysRevB.74.115315.

28. H.-T. Tan, W.-W. Deng and H. Huang, Multimode entanglement and squeezing from coupled four-wave mixing oscillators, J. Phys. B, At. Mol. Opt. Phys. 43 (2010) 215507, doi: https://doi.org/10.1088/0953-4075/43/21/215507.

29. W. Shi, F. Wang, L. Zhang, Z. Zhan and X. Li, Continuous-variable multimode entanglement in multi-wave mixing, Opt. Commun. 285 (2012) 4446-4452, doi: http://dx.doi. org/10.1016/j.optcom.2012.06.047.

30. T. C. H. Liew and V. Savona, Multimode entanglement in coupled cavity arrays, New J. Phys. 15 (2013) 025015, doi: https://doi.org/10.1088/1367-2630/15/2/025015.

31. P. A. Knott, T. J. Proctor, K. Nemoto, J. A. Dunningham and W. J. Munro, Effect of multimode entanglement on lossy optical quantum metrology, Phys. Rev. A 90 (2014) 033846, doi: https://doi.org/10.1103/PhysRevA.90.033846.

32. L. Marinatto and T. Weber, Teleportation with indistinguishable particles, Phys. Lett. A 287 (2001) 1, doi: http://dx.doi.org/10.1016/S0375-9601(01)00431-5.

33. S. P. Kulik, S. N. Molotkov and S. S. Straupe, On teleportation in a system of identical particles, JETP Lett. 92(3) (2010) 188, doi: 10.1134/S0021364010150130.

34. U. Marzolino and A. Buchleitner, Performances and robustness of quantum teleportation with identical particles, Proc. R. Soc. A 472 (2016) 20150621, doi: https://doi.org/ 10.1098/rspa.2015.0621.

35. D. J. Griffiths, Introduction to Elementary Particles, 2nd edn. (Wiley-VCH, Germany, 2008). 
36. D. H. Perkins, Introduction to High Energy Physics, 4th edn. (Cambridge University Press, Cambridge, 2000).

37. L. M. Krauss, The Physics of Star Trek, Reissue edn. (Flamingo, USA, 1995).

38. M. Dine and A. Kusenko, Origin of the matter-antimatter asymmetry, Rev. Mod. Phys. 76 (2003) 1, doi: https://doi.org/10.1103/RevModPhys.76.1.

39. M. E. Peskin and D. V. Schroeder, An Introduction to Quantum Field Theory (AddisonWesley, US, 1995).

40. Y. Aharonov and L. Susskind, Charge superselection rule, Phys. Rev. 155 (1967) 1428, doi: https://doi.org/10.1103/PhysRev.155.1428.

41. W. B. Rolnick, Does charge obey a superselection rule? Phys. Rev. Lett. 19 (1967) 717, doi: https://doi.org/10.1103/PhysRevLett.19.717.

42. V. Mourik, K. Zuo, S. M. Frolov, S. R. Plissard, E. P. A. M. Bakkers and L. P. Kouwenhoven, Signatures of Majorana fermions in hybrid superconductor-semiconductor nanowire devices, Science 336(6084) (2012) 1003-1007, doi: 10.1126/science.1222360.

43. S. Nadj-Perge, I. K. Drozdov, J. Li, H. Chen, S. Jeon, J. Seo, A. H. MacDonald, B. A. Bernevig and A. Yazdani, Observation of Majorana fermions in ferromagnetic atomic chains on a superconductor, Science 346(6209) (2014) 602-607, doi: 10.1126/ science.1259327.

44. M. T. Deng, S. Vaitiekenas, E. B. Hansen, J. Danon, M. Leijnse, K. Flensberg, J. Nygard, P. Krogstrup and C. M. Marcus, Majorana bound state in a coupled quantum-dot hybridnanowire system, Science 354(6319) (2016) 1557-1562, doi: 10.1126/science.aaf3961.

45. Q. L. He, L. Pan, A. L. Stern, E. C. Burks, X. Che, G. Yin and J. Wang, Chiral Majorana fermion modes in a quantum anomalous Hall insulator-superconductor structure, Science 357(6348) (2017) 294-299, doi: 10.1126/science.aag2792.

46. D. Zwillinger, CRC Standard Mathematical Tables and Formulae, 32nd edn. (CRC Press, US, 2011).

47. D. M. Greenberger, M. A. Horne and A. Zeilinger, Going Beyond Bell's Theorem, arXiv:0712.0921, https://arxiv.org/abs/0712.0921.

48. J. M. Leinaas, J. Myrheim and E. Ovrum, Geometrical aspects of entanglement, Phys. Rev. A 74 (2006) 012313, doi: https://doi.org/10.1103/PhysRevA.74.012313.

49. E. Klempt, C. Batty and J.-M. Richard, The antinucleon-nucleon interaction at low energy: Annihilation dynamics, Phys. Rep. 413(4-5) (2005) 197-317, doi: http://dx.doi. org/10.1016/j.physrep.2005.03.002.

50. M. Schlosshauer, Decoherence, the measurement problem, and interpretations of quantum mechanics, Rev. Mod. Phys. 76 (2005) 1267-1305, doi: https://doi.org/10.1103/ RevModPhys.76.1267.

51. D. McMahon, Quantum Computing Explained, 1st edn. (Wiley-IEEE Computer Society Pr, US, 2007).

52. Z. Zhao, Y.-A. Chen, A.-N. Zhang, T. Yang, H. J. Briegel and J.-W. Pan, Experimental demonstration of five-photon entanglement and open-destination teleportation, Nature 430 (2004) 54-58, doi: 10.1038/nature02643.

53. W. Dür and J. I. Cirac, Multiparty teleportation, J. Mod. Opt. 47(2-3) (2000) 247-255, doi: http://dx.doi.org/10.1080/09500340008244039.

54. M. Zukowski, A. Zeilinger, M. A. Horne and A. K. Ekert, "Event-ready-detectors" Bell experiment via entanglement swapping, Phys. Rev. Lett. 71 (1993) 4287, doi: https://doi. org/10.1103/PhysRevLett.71.4287.

55. C. Schmid, N. Kiesel, U. K. Weber, R. Ursin, A. Zeilinger and H. Weinfurter, Quantum teleportation and entanglement swapping with linear optics logic gates, New J. Phys. 11 (2009) 033008, doi: https://doi.org/10.1088/0031-8949/11/3/033008. 
56. D. E. Morrissey and M. J. Ramsey-Musolf, Electroweak baryogenesis, New J. Phys. 14 (2012) 125003, doi: https://doi.org/10.1088/1367-2630/14/12/125003.

57. S. Weinberg, Cosmology (Oxford University Press, New York, 2008).

58. A. Liddle, An Introduction to Modern Cosmology, 3rd edn. (Wiley, West Sussex, England, 2015).

59. L. Canetti, M. Drewes and M. Shaposhnikov, Matter and antimatter in the universe, New J. Phys. 14 (2012) 095012, doi: https://doi.org/10.1088/1367-2630/14/9/095012.

60. S. P. Ahlen, S. Barwick, J. J. Beatty, C. R. Bower, G. Gerbier, R. M. Heinz, D. Lowder, S. McKee, S. Mufson, J. A. Musser, P. B. Price, M. H. Salamon, G. Tarle, A. Tomasch and B. Zhou, New limit on the low-energy antiproton/proton ratio in the galactic cosmic radiation, Phys. Rev. Lett. 61 (1988) 145, doi: https://doi.org/10.1103/PhysRevLett.61.145.

61. A. G. Cohen, A. De Rujula and S. L. Glashow, A matter-antimatter universe? Astrophys. J. 495 (1998) 539, doi: 10.1086/305328.

62. G. Steigman, The radio continuum morphology of spiral galaxies, Annu. Rev. Astron. Astrophys. 14 (1976) 336, doi: 10.1146/annurev.aa.14.090176.002221.

63. P. A. M. Dirac, The quantum theory of the electron, Proc. R. Soc. Lond. A 117(778) (1928) 610-624, doi: 10.1098/rspa.1928.0023; The quantum theory of the electron. Part II, Proc. R. Soc. Lond. A 118(779) (1928) 351-361, doi: 10.1098/rspa.1928.0056; A theory of electrons and protons, Proc. R. Soc. Lond. A 126(801) (1930) 360-365, doi: 10.1098/rspa.1930.0013.

64. A. Kusenko, L. Pearce and L. Yang, Postinflationary higgs relaxation and the origin of matter-antimatter asymmetry, Phys. Rev. Lett. 114 (2015) 061302, doi: https://doi.org/ 10.1103/PhysRevLett.114.061302.

65. V. Kuzmin, V. Rubakov and M. Shaposhnikov, On anomalous electroweak baryonnumber non-conservation in the early universe, Phys. Lett. B 155 (1985) 36, doi: http:// dx.doi.org/10.1016/0370-2693(85)91028-7.

66. A. Dolgov, Non-GUT baryogenesis, Phys. Rep. 222 (1992) 309, doi: http://dx.doi.org/ 10.1016/0370-1573(92)90107-B.

67. M. Fukugita and T. Yanagida, Barygenesis without grand unification, Phys. Lett. B 174 (1986) 45, http://dx.doi.org/10.1016/0370-2693(86)91126-3.

68. S. Davidson, E. Nardi and Y. Nir, Phys. Rept. 466 (2008) 105-177, doi: http://dx.doi. org/10.1016/j.physrep.2008.06.002.

69. I. Affleck and M. Dine, A new mechanism for baryogenesis, Nucl. Phys. B 249 (1985) 361, doi: http://dx.doi.org/10.1016/0550-3213(85)90021-5.

70. S. Weinberg, Cosmological production of baryons, Phys. Rev. Lett. 42 (1979) 850, doi: https://doi.org/10.1103/PhysRevLett.42.850.

71. E. W. Kolb and M. S. Turner, The Early Universe (Adddison-Wesley, Reading, MA, 1990).

72. A. D. Sakharov, Violation of CP invariance, C asymmetry, and baryon asymmetry of the universe, JETP Lett. 5 (1967) 24-27; republished as A. D. Sakharov, Sov. Phys. Usp. 34(5) (1991) 392-393, doi: https://doi.org/10.1070/PU1991v034n05ABEH002497.

73. E. Komatsu et al., Seven-year Wilkinson microwave anisotropy probe (WMAP) observations: Cosmological interpretation, Astrophys. J. Suppl. 192 (2011) 18, doi: https:// doi.org/10.1088/0067-0049/192/2/18.

74. R. Dawkins, Universal Darwinism, in Evolution From Molecules to Man, ed. D. S. Bendall, 1st edn. (Cambridge University Press, Cambridge, 1983).

75. L. Smolin, The Life of the Cosmos (Oxford University Press, UK, 1997).

76. W. H. Zurek, Quantum Darwinism, Nat. Phys. 5 (2009) 181-188, doi: 10.1038/ nphys1202. 
77. J. J. Sakurai and J. Napolitano, Modern Quantum Mechanics (Addison-Wesley, Second Edition, San Francisco, 2011).

78. J. Yin, Y. Cao, H.-L. Yong, J.-G. Ren, H. Liang, S.-K. Liao, F. Zhou, C. Liu, Y.-P. Wu, G.-S. Pan, Q. Zhang, C.-Z. Peng and J.-W. Pan, Lower bound on the speed of nonlocal correlations without locality and measurement choice loopholes, Phys. Rev. Lett. 110 (2013) 260407, doi: https://doi.org/10.1103/PhysRevLett.110.260407.

79. G. B. Saha, Basics of PET Imaging: Physics, Chemistry, and Regulations, 2nd edn. (Springer, New York, 2010).

80. A. Vilenkin, Birth of inflationary universes, Phys. Rev. D 27 (1983) 2848, doi: https:// doi.org/10.1103/PhysRevD.27.2848.

81. A. D. Linde, Eternally existing self-reproducing chaotic inflanationary universe, Phys. Lett. B 175 (1986) 395-400, doi: http://dx.doi.org/10.1016/0370-2693(86)90611-8.

82. M. Tegmark, Parallel universes, Sci. Am. 288(5) (2003) 40-51, doi: 10.1038/scientificamerican0503-40. 